\title{
Multivariable Regulation in Geometric Terms: Old and New Results
}

\author{
Giovanni Marro*
}

\begin{abstract}
The aim of this lecture is to present some well-known and some new results in multivariable regulation in a strictly geometric framework, each compared with the corresponding singlevariable result approached with the standard transfer function techniques. It consists of three parts, each necessary for a background for the subsequent one: a selection of the basic tools, a survey of the solution of the asymptotically robust autonomous regulator problem with the internal model of the exosystem, and a presentation of the "steering along zeros technique" to obtain multivariable perfect tracking in the minimum-phase case or almost perfect tracking by using preaction in the nonminimum-phase case.
\end{abstract}

\section{Introduction}

The Geometric State-Space Theory is a collection of mathematical concepts developed to achieve a better and neater insight into the most salient features of multivariable linear dynamical systems in connection with compensator and regulator synthesis problems. It is based on the state space representation and is also very useful to easily link SISO and MIMO systems and to clarify in quite a concise and elegant way some common properties that cannot be pointed out by the transform-based techniques usually adopted in the SISO case. Although the geometric tools are very simple and supported by exhaustive computational machinery, it is rather difficult to get a complete panorama of them, since their presentation in the literature by several authors over a period of more than 25 years is not uniform in style and has very often been covered in unnecessarily heavy mathematics.

At the end of the sixties (1969) Basile, Marro and Laschi published some results using geometric techniques in five papers, three in Italian and two in English, that analyzed the basic tools and presented solutions to some problems to which they could be profitably applied: disturbance rejection [6], unknown-input observability [5, 35] and noninteraction [3]. The first of the two papers in English [4] presented definitions and properties of the major protagonists of this approach, that were called "controlled and conditioned invariants". At abouth the same time Wonham and Morse applied an algorithm similar to that for the maximal controlled invariant computation for the solution of noninteracting control problems [57]. In their paper only the algorithm was presented, while the name "invariant with respect to $(\mathrm{A}, \mathrm{B})$ ", to be later transformed into "(A,B)-invariant", was only introduced by the same authors abouttwo years afterwards [44].

\footnotetext{
${ }^{*}$ Dipartimento di Elettronica, Informatica e Sistemistica, Università di Bologna, Viale Risorgimento 2, I-40136 Bologna, Italy, Phone: +39 -51-2093046, Fax: +39 -51-2093073, E-Mail: gmarro@deis.unibo.it
} 
However, the work of Wonham and Morse during the seventies, in many cases in cooperation with Francis and Pearson [26, 27, 28, 54, 56, 58], was conclusive to investigate and propagate the use of the geometric techniques, in particular with reference to multivariable tracking and regulation problems. Wonham's book [55] is still an exhaustive standard reference for this approach.

In the eighties major contributions are due to Willems, with the theory of almost controlled and almost conditioned invariant subspaces to deal with high-gain feedback problems [51, 52], Willems and Commault [53] and Schumacher [47], who contributed with a complete study of the regulation problem, including structural disturbance rejection. The use of self-bounded controlled and self-hidden conditioned invariant subspaces by Basile and Marro [9, 12, 13] allowed for a more direct presentation of these results. Introduction of the robust controlled invariant, also by Basile and Marro [10], opened the way to new applications, for instance the elimination of regulation transients in multivariable plants under parameter jumps or configuration changes, recently approached by Marro and Piazzi [39].

This lecture consists of three sections. The purpose of the first is to present, in the simplest possible terms, a selection of the basic tools of the geometric approach, strictly necessary to discuss multivariable tracking and regulation. The tools are: controlled and conditioned invariants, self-bounded controlled invariants, system invertibility, perfect output controllability, constrained output controllability (multivariable relative degree), and invariant zeros. Since this selection is strictly oriented to treatment of tracking and regulation, it does not include some other tools, like almost controlled and conditioned invariants and the robust controlled invariant. Then, referring to the classical disturbance rejection problem with stability, it is shown that the geometric solvability conditions usually consist of two parts: a structural condition and a stabilizability condition; the latter can be stated both in terms of self-bounded controlled invariants or in terms of invariant zeros, and can be relaxed if there is some preview of the signal to reject.

The second section deals with the central points of feedback regulation: inclusion of an internal model of the exosystem in the regulator loop, and achievement of the closed-loop pole assignment: the SISO solution, that can be based on the Diophantine equation, is compared with the MIMO solution with the geometric techniques, and robustness of MIMO regulation, that requires as many replicas of the internal model as the number of regulated outputs, is briefly discussed.

The third section introduces perfect tracking as a further requirement for the previously derived asymptotic regulator and resorts to feedforward as a second degree-of-freedom feature. A solution with the standard transfer function analysis for the SISO case and the corresponding geometric solution for the MIMO case are presented, in which a special feedforward unit, that reproduces an approximate replica of the reference function by switching an exosystem and suitably filtering the obtained signal, is proposed to obtain perfect or almost perfect tracking also in the nonminimum-phase case. 


\section{The Tools}

In this section a brief review of the basic geometric tools is presented. By "basic" we understand:

1. strictly necessary to state a self-contained mathematical background for the theory of multivariable regulation and tracking presented in next sections;

2. strictly necessary to support the computational framework for the constructive numerical solution of all the presented problems.

They are: invariants, controlled invariants and self-bounded controlled invariants, conditioned invariants, system invertibility and functional output controllability, invariant zeros.

Let us note incidentally that the geometric tools are not only used for a neat presentation of multivariable control theory, but also provide a useful computational framework, based on few algorithms that extend some basic straightforward computations of the standard statespace approach to system theory (controllability, observability and pole assignment). Hence, the selection of the basic tools described in this section is also related to their impact on the computational framework.

The following notation is used. $\mathbf{R}$ stands for the field of real numbers, $\mathbf{C}$ for that of complex numbers, split into $\mathbf{C}_{-}, \mathbf{C}_{0}$ and $\mathbf{C}_{+}$(left half-plane, imaginary axis and right half-plane). Sets, vector spaces and subspaces are denoted by script capitals like $\mathcal{X}, \mathcal{I}, \mathcal{V}$, etc.; since most of the geometric theory of dynamic system herein presented is developed in the vector space $\mathbf{R}^{n}$, we reserve the symbol $\mathcal{X}$ for the full space, i.e., we assume $\mathcal{X}:=\mathbf{R}^{n}$. The orthogonal complement of any subspace $\mathcal{Y} \subseteq \mathcal{X}$ is denoted by $\mathcal{Y}^{\perp}$, matrices and linear maps by slanted capitals like $A$, $B$, etc., the image and the null space of the generic matrix or linear transformation $A$ by $\operatorname{im} A$ and $\operatorname{ker} A$ respectively, the transpose of the generic real matrix $A$ by $A^{T}$, the spectrum of $A$ by $\sigma(A)$, the $n \times n$ identity matrix by $I_{n}$.

The algorithms of the geometric approach require computations involving subspaces. A generic subspace $\mathcal{X}_{1} \subseteq \mathcal{X}$ is numerically defined with a basis matrix, i.e., a matrix $X_{1}$ having maximal rank such that $\mathcal{X}_{1}=\operatorname{im} X_{1}$. The operations on subspaces that are required in the geometric-type algorithms are the sum $\mathcal{X}_{1}+\mathcal{X}_{2}$, the orthogonal complementation $\mathcal{X}_{1}^{\perp}$, the intersection $\mathcal{X}_{1} \cap \mathcal{X}_{2}=\left(\mathcal{X}_{1}^{\perp}+\mathcal{X}_{2}^{\perp}\right)^{\perp}$, the direct linear mapping $A \mathcal{X}_{1}$ (where $A: \mathcal{X} \rightarrow \mathcal{X}$ denotes a linear map represented by the $n \times n$ matrix $A$ with respect to the main basis of $\mathcal{X}$ ), the inverse linear mapping $A^{-1} \mathcal{X}_{1}=\left(A^{T} \mathcal{X}_{1}^{\perp}\right)^{\perp}$.

The linear dynamic system herein considered as the reference to introduce the geometric tools is

$$
\begin{aligned}
\dot{x}(t) & =A x(t)+B u(t), \quad x(0)=x_{0} \\
y(t) & =C x(t)
\end{aligned}
$$

where $x \in \mathcal{X}\left(=\mathbf{R}^{n}\right), u \in \mathcal{U}\left(=\mathbf{R}^{p}\right)$ and $y \in \mathcal{Y}\left(=\mathbf{R}^{q}\right)$ denote respectively the state, input and output. Let $\mathcal{B}:=\operatorname{im} B$ and $\mathcal{C}:=\operatorname{ker} C$. System (2.1)-(2.2) is simply called the triple $(A, B, C)$, while the sole differential equation (2.1), describing the state evolution caused by the input $u(t)$ with $x_{0}=0$, is called the pair $(A, B)$, and the set consisting of equation (2.2) and equation (2.1) with only the first term on the right, describing the state evolution due to the initial state $x_{0}$, is called the pair $(C, A)$. Let us also recall that the two basic algebraic feedback connection considered in synthesis procedures, that are state-to-input through a matrix $F$, that transforms 
the triple $(A, B, C)$ into $(A+B F, B, C)$, and output-to-state through a matrix $G$, that produces $(A+G C, B, C)$, are called state feedback and output injection respectively.

\subsection{Invariants and Restricted Maps}

Definition 2.1 (Invariant) Given a linear map $A: \mathcal{X} \rightarrow \mathcal{X}$, a subspace $\mathcal{L} \subseteq \mathcal{X}$ is an $A$-invariant (or, simply, an invariant when the map referred to is clear from the context) if

$$
A \mathcal{L} \subseteq \mathcal{L}
$$

Let $L$ be a basis matrix of $\mathcal{L}$ : the following statements are equivalent to $(2.3)$ :

- a matrix $X$ exists such that $A L=L X$;

- $\mathcal{L}$ is a locus of trajectories of the free system $\dot{x}(t)=A x(t)$.

Given the subspaces $\mathcal{D}, \mathcal{E}$ contained in $\mathcal{X}$ and such that $\mathcal{D} \subseteq \mathcal{E}$, and a linear map $A: \mathcal{X} \rightarrow \mathcal{X}$, it is well known that the set of all $A$-invariants $\mathcal{L}$ satisfying $\mathcal{D} \subseteq \mathcal{L} \subseteq \mathcal{E}$ is a nondistributive lattice with respect to $\subseteq,+, \cap$. We denote with $\max \mathcal{L}(A, \mathcal{E})$ the maximal $A$-invariant contained in $\mathcal{E}$ (the sum of all the $A$-invariants contained in $\mathcal{E}$ ) and with $\min \mathcal{L}(A, \mathcal{D})$ the minimal $A$-invariant containing $\mathcal{D}$ (the intersection of all the $A$-invariants containing $\mathcal{D}$ ): the above lattice is nonempty if and only if $\mathcal{D} \subseteq \max \mathcal{L}(A, \mathcal{E})$ or $\min \mathcal{L}(A, \mathcal{D}) \subseteq \mathcal{E}$.

The restriction of map $A$ to the $A$-invariant subspace $\mathcal{L}$ is denoted by $\left.A\right|_{\mathcal{L}} ; \mathcal{L}$ is said to be internally stable if $\left.A\right|_{\mathcal{L}}$ is stable. Given two $A$-invariants $\mathcal{L}_{1}$ and $\mathcal{L}_{2}$ such that $\mathcal{L}_{1} \subseteq \mathcal{L}_{2}$, the map induced by $A$ on the quotient space $\mathcal{L}_{2} / \mathcal{L}_{1}$ is denoted by $\left.A\right|_{\mathcal{L}_{2} / \mathcal{L}_{1}}$. In particular, an $A$-invariant $\mathcal{L}$ is said to be externally stable if $\left.A\right|_{\mathcal{X} / \mathcal{L}}$ is stable.

Definition 2.2 (Complementable Invariant) $A n A$-invariant $\mathcal{L} \subseteq \mathcal{X}$ is said to be complementable if an $A$-invariant $\mathcal{L}_{c}$ exixts such that $\mathcal{L} \oplus \mathcal{L}_{c}=\mathcal{X}$; if so, $\mathcal{L}_{c}$ is called a complement of $\mathcal{L}$.

The importance of invariants in connection with the triple $(A, B, C)$ is related to the following well known, basic properties:

Property 2.1 The reachability subspace of the pair $(A, B)$, i.e., the set of all the states that can be reached from the origin in any finite time by means of admissible (piecewise continuous) control actions, is $\mathcal{R}=\min \mathcal{L}(A, \mathcal{B})$. If $\mathcal{R}=\mathcal{X},(A, B)$ is said to be reachable.

Property 2.2 The pair $(A, B)$ is pole-assignable with state feedback if and only if $\mathcal{R}=\mathcal{X}$, stabilizable with state feedback if and only if $\mathcal{R}$ is externally stable.

Property 2.3 The unobservability subspace of the pair $(C, A)$, i.e., the set of all the initial states that cannot be recognized from the output function, is $\mathcal{Q}=\max \mathcal{L}(A, \mathcal{C})$. If $\mathcal{Q}=\{0\},(C, A)$ is said to be observable.

Property 2.4 The pair $(C, A)$ is pole-assignable with output injection if and only if $\mathcal{Q}=\{0\}$, stabilizable with output injection if and only if $\mathcal{Q}$ is internally stable. 
Algorithm 2.1 (Minimal $A$-invariant containing $\mathcal{B})$ The subspace $\min \mathcal{L}(A, \mathcal{B})$ coincides with the last term of the sequence

$$
\begin{aligned}
& \mathcal{Z}_{0}:=\mathcal{B} \\
& \mathcal{Z}_{i}:=\mathcal{B}+A \mathcal{Z}_{i-1} \quad(i=1, \ldots, k)
\end{aligned}
$$

where the value of $k \leq n-1$ is determined by condition $\mathcal{Z}_{k+1}=\mathcal{Z}_{k}$.

Algorithm 2.2 (Maximal $A$-invariant contained in $\mathcal{C}$ ) The subspace $\max \mathcal{L}(A, \mathcal{C})$ satisfies

$$
\max \mathcal{L}(A, \mathcal{C})=\min \mathcal{L}\left(A^{T}, \mathcal{C}^{\perp}\right)^{\perp}
$$

hence it can be computed by using Algorithm 2.1 again.

Algorithm 2.3 (Internal and External Eigenstructure of an $A$-invariant) Matrices $P$ and $Q$ representing $\left.A\right|_{\mathcal{L}}$ and $\left.A\right|_{\mathcal{X} / \mathcal{L}}$ up to an isomorphism, are derived as follows. Let us consider the similarity transformation $T:=\left[L T_{2}\right]$, with $\operatorname{im} L=\mathcal{L}(L$ is a basis matrix of $\mathcal{L})$ and $T_{2}$ such that $T$ is nonsingular. In the new basis the linear transformation $A$ is expressed by

$$
A^{\prime}=T^{-1} A T=\left[\begin{array}{cc}
A_{11}^{\prime} & A_{12}^{\prime} \\
O & A_{22}^{\prime}
\end{array}\right]
$$

The requested matrices are defined as $P:=A_{11}^{\prime}, Q:=A_{22}^{\prime}$.

Algorithm 2.4 (Complementability of an A-invariant) Let us consider again the change of basis introduced in Algorithm 2.3. $\mathcal{L}$ is complementable if and only if the Sylvester equation ${ }^{1}$ in $X$

$$
A_{11}^{\prime} X-X A_{22}^{\prime}=-A_{12}^{\prime}
$$

admits a solution. If so, a basis matrix of $\mathcal{L}_{c}$ is given by $L_{c}:=L X+T_{2}{ }^{2}$

The following property states a sufficient condition for complementability that is often applied in regulation theory. In fact, if this condition is satisfied, equation (2.9) admits a unique solution.

Property 2.5 An A-invariant $\mathcal{L}$ is complementable and admits a unique complement $\mathcal{L}_{c}$ if the linear mappings $\left.A\right|_{\mathcal{L}}$ and $\left.A\right|_{\mathcal{X} / \mathcal{L}}$ (hence matrices $P$ and $Q$ provided by Algorithm 2.3) have no common eigenvalues, i.e., if

$$
\sigma\left(\left.A\right|_{\mathcal{L}}\right) \cap \sigma\left(\left.A\right|_{\mathcal{X} / \mathcal{L}}\right)=\emptyset
$$

${ }^{1}$ The generic Sylvester equation $A X+X B=C$, where $A$ is $m \times m, B$ is $n \times n$ and $X, C$ are both $m \times n$, is equivalent to a set of $m n$ linear equation. It has a unique solution if $\sigma(A) \cap \sigma(B)=\emptyset$.

${ }^{2}$ A sketch of the proof. Let $m$ be the dimension of $\mathcal{L}$. In the new coordinate system the matrices

$$
L^{\prime}=\left[\begin{array}{c}
I_{m} \\
O
\end{array}\right] \quad \text { and } \quad L_{c}^{\prime}=\left[\begin{array}{c}
X \\
I_{n-m}
\end{array}\right]
$$

are basis matrices of $\mathcal{L}$ and a complement $\mathcal{L}_{c}$ respectively, since $\left[L^{\prime} L_{c}^{\prime}\right]$ is clearly nonsingular and both satisfy (2.4). The corresponding basis matrices in the original basis are $T L^{\prime}$ and $T L_{c}^{\prime}$. 


\subsection{Controlled Invariants and Self-Bounded Controlled Invariants}

Definition 2.3 (Controlled Invariant) Given a linear map $A: \mathcal{X} \rightarrow \mathcal{X}$ and a subspace $\mathcal{B} \subseteq \mathcal{X}$, a subspace $\mathcal{V} \subseteq \mathcal{X}$ is an $(A, \mathcal{B})$-controlled invariant (or, simply, a controlled invariant if $A$ and $\mathcal{B}$ are clear from the context) if

$$
A \mathcal{V} \subseteq \mathcal{V}+\mathcal{B}
$$

Let $B$ and $V$ be basis matrices of $\mathcal{B}$ and $\mathcal{V}$ respectively: the following statements are equivalent to (2.11):

- a matrix $F$ exists such that $(A+B F) \mathcal{V} \subseteq \mathcal{V}$;

- matrices $X$ and $U$ exist such that $A V=V X+B U$;

$-\mathcal{V}$ is a locus of trajectories of the pair $(A, B)$.

The last statement is very important in connection with control problems: a subspace $\mathcal{V} \subseteq \mathcal{X}$ is an $(A, \mathcal{B})$-controlled invariant if and only if, starting from any initial state belonging to $\mathcal{V}$, it is possible to follow a state trajectory completely belonging to $\mathcal{V}$.

It is easily shown that the sum of any two controlled invariants is a controlled invariant; thus the set of all controlled invariants contained in a given subspace $\mathcal{E} \subseteq \mathcal{X}$ is a semilattice with respect to $\subseteq,+$, hence admits a supremum, the maximal $(A, \mathcal{B})$-controlled invariant contained in $\mathcal{E}$, that is denoted by $\max \mathcal{V}(A, \mathcal{B}, \mathcal{E})$ (or simply $\mathcal{V}^{*}$ if the matrices and subspaces involved in the definition are clear from the context).

Referring to the pair $(A, B)$, we denote with $\mathcal{R}_{\mathcal{V}}$ the reachable subspace from the origin by trajectories constrained to belong to $\mathcal{V}$. By virtue of $(2.12)$ it is derived as $\mathcal{R}_{\mathcal{V}}=$ $\min \mathcal{L}(A+B F, \mathcal{V} \cap \mathcal{B})$ and, being clearly an $(A+B F)$-invariant, it also is an $(A, \mathcal{B})$-controlled invariant.

A generic $(A, \mathcal{B})$-controlled invariant $\mathcal{V}$ is said to be internally stabilizable or externally stabilizable if at least one matrix $F$ exists such that $\left.(A+B F)\right|_{\mathcal{V}}$ is stable or at least one matrix $F$ exists such that $\left.(A+B F)\right|_{\mathcal{X} / \mathcal{V}}$ is stable. It is easily proved that the eigenstructure of $\left.(A+B F)\right|_{\mathcal{V} / \mathcal{R}_{\mathcal{V}}}$ is independent of $F$; it is called the internal unassignable eigenstructure of $\mathcal{V}: \mathcal{V}$ is both internally and externally stabilizable with the same $F$ if and only if its internal unassignable eigenstructure is stable and the $A$-invariant $\mathcal{V}+\min \mathcal{L}(A, \mathcal{B})$ is externally stable. Hence, referring to the pair $(A, B)$, external stabilizability of any controlled invariant is guaranteed if $(A, B)$ is stabilizable: thus, under this assumption, we can state that any $(A, \mathcal{B})$-controlled invariant is stabilizable if and only if its internal unassignable eigenstructure is stable.

Definition 2.4 (Self-Bounded Controlled Invariant) Given a linear map $A: \mathcal{X} \rightarrow \mathcal{X}$ and two subspaces $\mathcal{B} \subseteq \mathcal{X}, \mathcal{E} \subseteq \mathcal{X}$, a subspace $\mathcal{V} \subseteq \mathcal{X}$ is an $(A, \mathcal{B})$-controlled invariant self-bounded with respect to $\mathcal{E}$ if, besides (2.11), the following relations hold

$$
\begin{aligned}
& \mathcal{V} \subseteq \mathcal{V}^{*} \subseteq \mathcal{E} \\
& \mathcal{V}^{*} \cap \mathcal{B} \subseteq \mathcal{V}
\end{aligned}
$$

The set of all $(A, \mathcal{B})$-controlled invariants self-bounded with respect to $\mathcal{E}$ is a nondistributive lattice with respect to $\subseteq,+, \cap$, whose supremum is $\mathcal{V}^{*}$ and whose infimum is $\mathcal{R}_{\mathcal{V}^{*}}$.

Given subspaces $\mathcal{D}, \mathcal{E}$ contained in $\mathcal{X}$ and such that $\mathcal{D} \subseteq \mathcal{V}^{*}$, the infimum of the lattice of all $(A, \mathcal{B})$-controlled invariants self-bounded with respect to $\mathcal{E}$ and containing $\mathcal{D}$ is the reachable 
set restricted to $\mathcal{V}^{*}$ with forcing action $\mathcal{B}+\mathcal{D}$, i.e., $\min \mathcal{L}\left(A+B F, \mathcal{V}^{*} \cap \mathcal{B}+\mathcal{D}\right)$, with $F$ such that $(A+B F) \mathcal{V}^{*} \subseteq \mathcal{V}^{*}$. The following property makes the concept of self-boundedness very interesting in connection with synthesis procedures with stability requirements.

Property 2.6 Let $\mathcal{D} \subseteq \mathcal{V}^{*}=\max \mathcal{V}(A, \mathcal{B}, \mathcal{E})$. If the infimum of the lattice of all $(A, \mathcal{B})$-controlled invariants self-bounded with respect to $\mathcal{E}$ and containing $\mathcal{D}$ is not internally stabilizable, no other $(A, \mathcal{B})$-controlled invariant contained in $\mathcal{E}$ and containing $\mathcal{D}$ is internally stabilizable.

\subsection{Conditioned Invariants}

Definition 2.5 (Conditioned Invariant) Given a linear map $A: \mathcal{X} \rightarrow \mathcal{X}$ and a subspace $\mathcal{C} \subseteq \mathcal{X}$ a subspace $\mathcal{S} \subseteq \mathcal{X}$ is an $(A, \mathcal{C})$-conditioned invariant (or, simply, a conditioned invariant when $A$ and $\mathcal{C}$ are clear from the context) if

$$
A(\mathcal{S} \cap \mathcal{C}) \subseteq \mathcal{S}
$$

Let $C$ be a matrix such that $\mathcal{C}=\operatorname{ker} C$. A statement equivalent to $(2.17)$ is:

- a matrix $G$ exists such that $(A+G C) \mathcal{S} \subseteq \mathcal{S}$.

The intersection of any two conditioned invariants is a conditioned invariant; thus the set of all conditioned invariants containing a given subspace $\mathcal{D} \subseteq \mathcal{X}$ is a semilattice with respect to $\subseteq, \cap$, hence admits an infimum, the minimal $(A, \mathcal{C})$-conditioned invariant containing $\mathcal{D}$, that is denoted by $\min \mathcal{S}(A, \mathcal{C}, \mathcal{D})$ (or simply $\mathcal{S}^{*}$ if the matrices and subspaces involved in the definition are clear from the context).

Controlled and conditioned invariants are dual each other. Controlled invariants are used in control problems, while conditioned invariants are used in observation problems. The orthogonal complement of an $(A, \mathcal{C})$-conditioned invariant is an $\left(A^{T}, \mathcal{C}^{\perp}\right)$-controlled invariant, hence the orthogonal complement of an $(A, \mathcal{C})$-conditioned invariant containing a given subspace $\mathcal{D}$ is an $\left(A^{T}, \mathcal{C}^{\perp}\right)$-controlled invariant contained in $\mathcal{D}^{\perp}$. Furthermore, the infimum of the lattice of all $(A, \mathcal{B})$-controlled invariants self-bounded with respect to a given subspace $\mathcal{E}$ can be expressed in terms of conditioned invariants as follows.

Property 2.7 Let $\mathcal{D} \subseteq \mathcal{V}^{*}=\max \mathcal{V}(A, \mathcal{B}, \mathcal{E})$. The infimum of the lattice of all $(A, \mathcal{B})$-controlled invariants self-bounded with respect to $\mathcal{E}$ and containing $\mathcal{D}$ is expressed by

$$
\max \mathcal{V}(A, \mathcal{B}, \mathcal{E}) \cap \min \mathcal{S}(A, \mathcal{E}, \mathcal{B}+\mathcal{D})
$$

Note, in particular, that $\mathcal{R}_{\mathcal{V}^{*}}=\mathcal{V}^{*} \cap \mathcal{S}^{*}$, with $S^{*}:=\min \mathcal{S}(A, \mathcal{E}, \mathcal{B})$.

Algorithm 2.5 (Minimal $(A, \mathcal{C})$-conditioned invariant containing $\mathcal{B}$ ) The subspace $S^{*}=$ $\min \mathcal{S}(A, \mathcal{C}, \mathcal{B})$ coincides with the last term of the sequence

$$
\begin{aligned}
\mathcal{Z}_{0} & :=\mathcal{B} \\
\mathcal{Z}_{i} & :=\mathcal{B}+A\left(\mathcal{Z}_{i-1} \cap \mathcal{C}\right) \quad(i=1, \ldots, k)
\end{aligned}
$$

where the value of $k \leq n-1$ is determined by condition $\mathcal{Z}_{k+1}=\mathcal{Z}_{k}$. 
Algorithm 2.6 (Maximal $(A, \mathcal{B})$-controlled invariant contained in $\mathcal{E}$ ) The subspace $\mathcal{V}^{*}=$ $\max \mathcal{V}(A, \mathcal{B}, \mathcal{E})$ satisfies

$$
\max \mathcal{V}(A, \mathcal{B}, \mathcal{E})=\min \mathcal{S}\left(A^{T}, \mathcal{B}^{\perp}, \mathcal{E}^{\perp}\right)^{\perp}
$$

hence it can be computed by using Algorithm 2.5.

Algorithm 2.7 (Matrix $F$ such that $(A+B F) \mathcal{V} \subseteq \mathcal{V})$ Let $V$ be a basis matrix of the $(A, \mathcal{B})$ controlled invariant $\mathcal{V}$. Matrices $X$ and $U$ satisfying (2.13) are derived with

$$
\left[\begin{array}{l}
X \\
U
\end{array}\right]=[V B]^{+} A V
$$

where the symbol ${ }^{+}$denotes the pseudoinverse. Then, assume

$$
F:=-U\left(V^{T} V\right)^{-1} V^{T}
$$

Matrix $F$ satisfies $(A+B F) V=V X$, hence, by the equivalence of (2.3) and (2.4), $(A+B F) \mathcal{V} \subseteq \mathcal{V}$.

Algorithm 2.8 (Internal unassignable eigenstructure of an $(A, \mathcal{B})$-controlled invariant) $A m a$ trix $P$ representing the map $\left.(A+B F)\right|_{\mathcal{V} / \mathcal{R}_{\mathcal{V}}}$ up to an isomorphism, is derived as follows. Let us consider the similarity transformation $T:=\left[\begin{array}{lll}T_{1} & T_{2} T_{3}\end{array}\right]$, with $\operatorname{im} T_{1}=\mathcal{R}_{\mathcal{V}}, \operatorname{im} T_{2}=\mathcal{V}$ and $T_{3}$ such that $T$ is nonsingular. In the new basis matrix $A+B F$ is expressed by

$$
(A+B F)^{\prime}=T^{-1}(A+B F) T=\left[\begin{array}{ccc}
A_{11}^{\prime} & A_{12}^{\prime} & A_{13}^{\prime} \\
O & A_{22}^{\prime} & A_{23}^{\prime} \\
O & O & A_{33}^{\prime}
\end{array}\right]
$$

The requested matrix is $P:=A_{22}^{\prime}$.

\subsection{System Invertibility and Perfect Output Controllability}

The concepts of system invertibility and output controllability of the triple $(A, B, C)$ are of paramount importance when approaching problems related to perfect tracking.

Definition 2.6 (Invertibility of a triple) Let us refer to the triple $(A, B, C)$, where $B$ is assumed to have maximum rank, and consider the linear operator $T_{f}: \mathcal{U}_{f} \rightarrow \mathcal{Y}_{f}$, from the vector space of the admissible input functions to the vector space of the zero-state responses, expressed by the convolution integral

$$
y(t)=C \int_{0}^{t} e^{A(t-\tau)} B u(\tau) d \tau
$$

$(A, B, C)$ is said to be invertible (or zero-state invertible)) if $\operatorname{ker} T_{f}=\{0\}$.

Let $\mathcal{V}^{*}:=\max \mathcal{V}(A, \mathcal{B}, \mathcal{C})$ with $\mathcal{B}:=\operatorname{im} B, \mathcal{C}:=\operatorname{ker} C$, and denote, as before, by $\mathcal{R}_{\mathcal{V}^{*}}$ the reachable subspace on $\mathcal{V}^{*}$ : clearly $\operatorname{ker} T_{f}=\{0\}$ if and only if $\mathcal{R}_{\mathcal{V}^{*}}=\{0\}$. Hence the following property holds. 
Property 2.8 The triple $(A, B, C)$, where $B$ is assumed to be maximum-rank, is invertible (zero-state invertible) $)^{3}$ if

$$
\mathcal{V}^{*} \cap \mathcal{B}=\{0\}
$$

Definition 2.7 (Functional Output Controllability) Refer to the operator (2.25) and denote by $\mathcal{Y}_{f}^{(n)}$ the set of all "smooth enough" output functions, i.e., of all output functions whose derivative of order $n$ or more is piecewise continuous. $(A, B, C)$ is said to be functionally output-controllable if $\mathcal{Y}_{f}^{(n)} \subseteq \operatorname{im} T_{f} \cdot{ }^{4}$

The following property is easily derived from Property 2.8 by a simple duality argument.

Property 2.9 Let $\mathcal{S}^{*}:=\min \mathcal{S}(A, \mathcal{C}, \mathcal{B})$ with $\mathcal{B}:=\operatorname{im} B, \mathcal{C}:=\operatorname{ker} C$. The triple $(A, B, C)$, where $C$ is assumed to be maximum-rank, is functionally output-controllable if

$$
\mathcal{S}^{*}+\mathcal{C}=\mathcal{X}
$$

In order to state a very neat extension to MIMO systems of the concept of relative degree that characterizes the output function smoothness in the SISO case, let us introduce the following extension of functional output controllability, that refers to a subspace of the output space and to the generic $h$-th derivative of the output function instead of the $n$-th derivative.

Definition 2.8 (Constrained Functional Output Controllability) A subspace $\mathcal{Y}^{(h)} \subseteq \mathbf{R}^{q}$ is said to be a functional output controllability subspace with respect to the $h$-th derivative if the output of the triple $(A, B, C)$ can be driven along any trajectory $y(\cdot)$ such that $y(t) \in \mathcal{Y}^{(h)}$ for all $t$ and with the $h$-th derivative piecewise continuous.

This is possible if and only if at least one $(A, \mathcal{B})$-controlled invariant $\mathcal{V} \subseteq \mathcal{X}$ exists such that $\mathcal{Y}^{(h)}=C \mathcal{V}$ and that, for every initial state $x_{0} \in \mathcal{V}$, it is possible to drive the state along a trajectory on $\mathcal{V}$ that maps into the given $y(\cdot)$ in the output space. The following characterizing property holds.

Property 2.10 Let us refer to $(A, B, C)$. An $(A, \mathcal{B})$-controlled invariant $\mathcal{V} \subseteq \mathcal{X}$ corresponds to a functional output controllability subspace with respect to the $h$-th derivative $\mathcal{Y}^{(h)}$ if

$$
\begin{aligned}
& \mathcal{Y}^{(h)}=C \mathcal{V} \\
& \mathcal{V} \subseteq \mathcal{V} \cap \mathcal{Z}_{h-1}+\mathcal{C}
\end{aligned}
$$

with $\mathcal{Z}_{h-1}$ defined by

$$
\begin{aligned}
& \mathcal{Z}_{0}:=\mathcal{B} \\
& \mathcal{Z}_{j}:=\mathcal{B}+A\left(\mathcal{Z}_{j-1} \cap \mathcal{V} \cap \mathcal{C}\right) \quad(j=1, \ldots, h-1)
\end{aligned}
$$

where, as before, $\mathcal{C}:=\operatorname{ker} C$ and $\mathcal{B}:=\operatorname{im} B$.

${ }^{3}$ A "strong" system invertibility refers to the linear operator $T_{f}^{\prime}: \mathcal{X} \times \mathcal{U}_{f} \rightarrow \mathcal{Y}_{f}$ defined by

$$
y(t)=C e^{A t} x_{0}+C \int_{0}^{t} e^{A(t-\tau)} B u(\tau) d \tau
$$

whose null space is clearly zero if and only if $\mathcal{V}^{*}=\{0\}$.

${ }^{4}$ It is worth noting that in the system theory literature zero-state invertible systems are often called leftinvertible ("left" since based on the kernel of the input-to-output functional map being zero) and zero-state functionally output-controllable systems are often called right-invertible ("right" since based on the image of the same functional map being the full space). 
Property 2.10 is consistent with Property 2.9: in fact, the whole output space $\mathbf{R}^{q}$ satisfies Property 2.10 with $h=n$ : in this case the stated conditions are satisfied with $\mathcal{V}=\mathcal{X}$, hence sequence (2.30) provides $\mathcal{S}^{*}$, so that $(2.29)$ is equivalent to $(2.27)$.

Algorithm 2.9 (Maximum Subspace of Constrained Functional Output Controllability) Let us refer to $(A, B, C)$. Given a subspace $\mathcal{Y}^{(h)} \subseteq \mathbf{R}^{q}$, let $\mathcal{E}:=C^{-1} \mathcal{Y}^{(h)}$; the maximal $(A, B)$-controlled invariant $\mathcal{V}_{\mathcal{E}}^{(h)} \subseteq \mathcal{X}$ contained in $\mathcal{E}$ such that the output can be driven on $C \mathcal{V}_{\mathcal{E}}^{(h)} \subseteq \mathcal{Y}^{(h)}$ along any trajectory $y(t)$ with piecewise continuous $h$-th derivative for all the initial states $x(0) \in \mathcal{V}_{\mathcal{E}}^{(h)}$, is the last term (with identity of two consecutive terms as the stop condition) of the sequence

$$
\begin{aligned}
& \mathcal{V}_{0}=\mathcal{E} \\
& \mathcal{V}_{i}=\max \mathcal{V}\left(A, \mathcal{B}, \mathcal{V}_{i-1} \cap \mathcal{Z}_{i, h-1}+\mathcal{C}\right) \quad(i=1,2, \ldots)
\end{aligned}
$$

with $\mathcal{Z}_{i, h-1}$ defined by the recursion process

$$
\begin{aligned}
& \mathcal{Z}_{i, 0}=\mathcal{B} \\
& \mathcal{Z}_{i, j}=\mathcal{B}+A\left(\mathcal{Z}_{i, j-1} \cap \mathcal{V}_{i-1} \cap \mathcal{C}\right) \quad(j=1, \ldots, h-1)
\end{aligned}
$$

where, as before, $\mathcal{C}:=\operatorname{ker} C$ and $\mathcal{B}:=\operatorname{im} B$.

Algorithm 2.10 (Multivariable Relative Degree) The relative degree $\rho_{i}$ of output $y_{i}$, $(i=1, \ldots, q)$ is obtained with Algorithm 2.9 by assuming $\mathcal{Y}^{(h)}:=\left\{y: y_{k}=0, k=1, \ldots, q, k \neq i\right\}$ (the $i$-th coordinate axis) and repeating for $h=1,2, \ldots$ until equality $\mathcal{Y}^{(h)}=C \mathcal{V}_{\mathcal{E}}^{(h)}$ is obtained. When this occurs, we have $\rho_{i}=h$.

\subsection{Invariant Zeros}

Invariant zeros are characteristic parameters of the triple $(A, B, C)$ that significantly affect solvability of both the standard asymptotic robust regulation problem, presented in Section 3, and the perfect tracking problem presented in Section 4. Roughly speaking, an invariant zero corresponds to a mode that, if suitably injected at the input of a dynamic system, can be nulled at the output by a suitable choice of the initial state. ${ }^{5}$

Definition 2.9 (Invariant Zero and Invariant Zero Structure) The invariant zeros of $(A, B, C)$ are the internal unassignable eigenvalues of $\mathcal{V}^{*}:=\max \mathcal{V}(A, \mathcal{B}, \mathcal{C})$. The invariant zero structure of $(A, B, C)$ is the internal unassignable eigenstructure of $\mathcal{V}^{*}$.

Thus, the invariant zeros are the eigenvalues of matrix $P$ derived with Algorithm 2.8, while the invariant zeros structure is the eigenstructure of matrix $P$ itself.

\footnotetext{
${ }^{5}$ This is particularly clear in the SISO case. In fact, the second term on the left of

$$
\frac{1}{s-z_{i}} \frac{K\left(s-z_{1}\right) \ldots\left(s-z_{m}\right)}{\left(s-p_{1}\right) \ldots\left(s-p_{n}\right)}-\frac{K\left(s-z_{1}\right) \ldots\left(s-z_{i-1}\right)\left(s-z_{i+1}\right) \ldots\left(s-z_{m}\right)}{\left(s-p_{1}\right) \ldots\left(s-p_{n}\right)}=0
$$

being a strictly proper rational function with all poles equal to those of the system, can be interpreted as the Laplace transform of a free motion. It is shown here by using a geometric-type argument that a similar property holds in the MIMO case, but the mode corresponding to any invariant zero must be distributed, with suitable coefficients, on all the inputs.
} 
Property 2.11 Let $W$ be a real $m \times m$ matrix having the invariant zero structure of $(A, B, C)$ as eigenstructure. A real $p \times m$ matrix $L$ and a real $n \times m$ matrix $X$ exist, with $(X, W)$ observable, such that applying to $(A, B, C)$ the input function

$$
u(t)=L e^{W t} v_{0}
$$

where $v_{0} \in \mathbf{R}^{m}$ denotes an arbitrary column vector, and starting from the initial state $x_{0}=X v_{0}$, the output $y(\cdot)$ is identically zero, while the state evolution (on $\operatorname{ker} C$ ) is described by

$$
x(t)=X e^{W t} v_{0}
$$

The observability of $(X, W)$ implies that all the modes corresponding to the zero structure can be excited with a suitable choice of $v_{0}$; since $(A, C)$ is observable and the output is identically zero all these modes are necessarily injected at the input through $L$.

Note that the application of the mode corresponding to a single zero is included in the statement; since $v_{0}$ is arbitrary, $W$ is defined within an isomorphism, hence it can be assumed to be in the real Jordan form without any loss of generality. Thus, the modes corresponding to its eigenvalues, both simple and multiple, can be easily individually excited by suitably setting to nonzero values only the components of $v_{0}$ that represent the initial conditions of these modes.

In simple words Property 2.11 means that any linear combination of the modes corresponding to the invariant zero structure, if suitably injected at the input, can be nulled at the output by a suitable choice of the initial state.

By substituting (2.33) and (2.34) in (2.1) and (2.2) the following equations for $L$ and $X$ are immediately obtained:

$$
\begin{aligned}
A X-X W & =-B L \\
C X & =O
\end{aligned}
$$

Algorithm 2.11 (Matrices $L$ and $X$ defined in Property 2.11) First compute a state feedback matrix $F$ such that $(A+B F) \mathcal{V}^{*} \subseteq \mathcal{V}^{*}$. $F$ also allows arbitrary assignment of all the eigenvalues except the internal unassignable eigenvalues of $\mathcal{V}^{*}$, so we can assume that no other eigenvalue of $A+B F$ is equal to them. On this assumption $\mathcal{R}_{\mathcal{V}^{*}}$ is an $(A+B F)$-invariant complementable with respect to $\mathcal{V}^{*}$ : hence an $(A, \mathcal{B})$-controlled invariant $\mathcal{V}$ exists such that $\mathcal{R}_{\mathcal{V}^{*}} \oplus \mathcal{V}=\mathcal{V}^{*},(A+B F) \mathcal{V} \subseteq \mathcal{V}$. Consider the change of basis defined by the transformation $T:=\left[T_{1} T_{2} T_{3}\right]$, with $\operatorname{im} T_{1}=\mathcal{R}_{\mathcal{V}^{*}}, \operatorname{im} T_{2}=\mathcal{V}$. With respect to the new basis we obtain the structure

$$
T^{-1}(A+B F) T=\left[\begin{array}{ccc}
A_{11}^{\prime} & O & A_{13}^{\prime} \\
O & A_{22}^{\prime} & A_{23}^{\prime} \\
O & O & A_{33}^{\prime}
\end{array}\right]
$$

Note that the invariant zeros are the eigenvalues of $A_{22}^{\prime}$ and the invariant zero structure is the eigenstructure of $A_{22}^{\prime}$. Then, assume $L:=F T_{2}, X:=T_{2}{ }^{6}$

\footnotetext{
${ }^{6}$ A sketch of the proof. Since $W$ is defined within an isomorphism, it is possible to assume $W=A_{22}^{\prime}$ without loss of generality, so that (2.35) becomes $A T_{2}-T_{2} A_{22}^{\prime}=-B F T_{2}$, or $(A+B F) T_{2}=T_{2} A_{22}^{\prime}$, that directly follows from (2.37). Since $\operatorname{im} X=\mathcal{V} \subseteq \mathcal{V}^{*},(2.36)$ is satisfied. Furthermore, the pair $(X, W)$ is observable since the rank of $X$ is maximal and equal to the dimension of $W$.
} 


\subsection{A classical application: the Disturbance Localization Problem}

The disturbance localization problem is one of the earliest (1969) applications of controlled invariants, and since it played the role of the reference problem for introducing new tools (like, for instance, self-bounded controlled invariants) it is here briefly recalled. Let us consider the system

$$
\begin{aligned}
\dot{x}(t) & =A x(t)+B u(t)+D d(t) \\
y(t) & =C x(t)
\end{aligned}
$$

where $u$ denotes the manipulable input, $d$ the disturbance input, that may be either inaccessible of accessible for measurement.

Problem 2.1 (Disturbance Localization) Referring to system (2.38), 2.39), determine, if possible, a state-to-input feedback matrix $F$, and, if disturbance is accessible, a disturbance-to-input matrix $S$, such that, starting at the zero state, any disturbance function $d(\cdot)$ does not affect the output $y$.

The system with state feedback is described by

$$
\begin{aligned}
\dot{x}(t) & =(A+B F) x(t)+D_{1} d(t) \\
y(t) & =C x(t)
\end{aligned}
$$

with $D_{1}:=D$ if disturbance is inaccessible or $D_{1}:=D+B S$, where $S$ is a suitable matrix to be determined, if disturbance is accessible. It behaves as requested if and only if its reachable set by $d$, i.e., the minimum $(A+B F)$-invariant containing $\mathcal{D}_{1}:=\operatorname{im} D_{1}$, is contained in $\mathcal{C}:=\operatorname{ker} C$.

Let $\mathcal{V}^{*}:=\max \mathcal{V}(A, \mathcal{B}, \mathcal{C})$. Since any $(A+B F)$-invariant is an $(A, \mathcal{B})$-controlled invariant, the inaccessible disturbance localization problem has a solution if and only if the following structural condition holds:

$$
\mathcal{D} \subseteq \mathcal{V}^{*}
$$

while in the case of the accessible disturbance localization problem, inclusion (2.42) is replaced by

$$
\mathcal{D} \subseteq \mathcal{V}^{*}+\mathcal{B}
$$

Conditions (2.42) and (2.43) are constructive in the sense that, if it they are satisfied, Algorithm 2.7 directly provides a matrix $F$ that solves the problem of inaccessible disturbance localization, while matrix $S$ is such that $D+B S$ is the projection of $d$ on $\mathcal{V}^{*}$ along $\mathcal{B}$. However, it is technically sound to require stability besides disturbance localization. The disturbance localization problem with stability is stated as follows.

Problem 2.2 (Disturbance Localization with Stability) Referring to system (2.38), (2.39), where $(A, B)$ is assumed to be stabilizable, determine, if possible, a state-to-input feedback matrix $F$, and, if disturbance is accessible, a disturbance-to-input matrix $S$, such that, starting at the zero state, any disturbance function $d(\cdot)$ does not affect the output $y$ and the system matrix $A+B F$ is strictly stable. 
Let us suppose that (2.42) or (2.43), clearly still necessary, are satisfied and denote by

$$
\mathcal{V}_{m}:=\mathcal{V}^{*} \cap \mathcal{S}^{*}=\max \mathcal{V}(A, \mathcal{B}, \mathcal{C}) \cap \min \mathcal{S}(A, \mathcal{C}, \mathcal{B}+\mathcal{D})
$$

the minimum controlled invariant self-bounded with respect to $\mathcal{C}$ and satisfying (2.42) or (2.43), that can be also computed by using the relation

$$
\mathcal{V}_{m}:=\min \mathcal{L}\left(A+B F, \mathcal{V}^{*} \cap(\mathcal{B}+\mathcal{D})\right) \text { with } F \text { such that }(A+B F) \mathcal{V}^{*} \subseteq \mathcal{V}^{*}
$$

From Property 2.6 the following statement is directly obtained.

Property 2.12 The disturbance localization problem with stability is solvable if and only if (2.43) or (2.43) holds and $\mathcal{V}_{m}$ is internally stabilizable.

Note that solvability of the problem is expressed by two types of conditions: the first is a structural condition and the second a stabilizability condition. This feature is also exhibited by the other synthesis problems approached in the next sections of this monography. The stabilizability condition can be expressed in terms of invariant zeros as follows.

Property 2.13 Let $\mathcal{Z}(u ; y)$ be the set of all the invariant zeros of $(38,39)$ referred to input $u$ and $\mathcal{Z}(u, d ; y)$ that referred to inputs $u, d$, considered as a whole. The disturbance localization problem with stability is solvable if and only if (2.42) or (2.43) holds and the elements of $\mathcal{Z}(u ; y)$ not belonging also to $\mathcal{Z}(u, d ; y)$ are all stable.

Property 2.13 is derived from Property 2.12 as follows: let $\mathcal{R}_{\mathcal{V}^{*}}$ be the reachable set on $\mathcal{V}^{*}$ by $u$, while $\mathcal{V}_{m}$ coincides with the reachable set on $\mathcal{V}^{*}$ by $u$ and $d$, both considered as manipulable inputs; both are clearly $(A+B F)$-invariants. The internal unassignable eigenvalues of $\mathcal{V}_{m}$ are the elements of $\sigma\left(A+\left.B F\right|_{\mathcal{V}_{m} / \mathcal{R}_{\mathcal{V}^{*}}}\right)$, while the set of zeros considered in the statement are defined by $\mathcal{Z}(u ; y):=\sigma\left(A+\left.B F\right|_{\mathcal{V}^{*} / \mathcal{R}_{\mathcal{V}^{*}}}\right)$ and $\left.\mathcal{Z}(u, d ; y):=\left.\sigma(A+B F)\right|_{\mathcal{V}^{*} / \mathcal{R}_{\mathcal{V}_{m}}}\right)$.

The main criticism to which the geometric techniques are subject is that they generally provide non-robust solutions. The disturbance localization problem is emblematic with respect to this: small variations of the controlled system parameters may destroy the structural feature consisting of a very vulnerable subspace inclusion.

In the next section it is shown that robust multivariable disturbance rejection can be achieved by feedback and studied again with geometric techniques, but within relaxed specifications: disturbance is modelled by an exosystem (reproducing, for instance, a step) and asymptotic robust rejection is achieved with a suitable feedback regulator. Since the error dynamics is arbitrary under standard controllability and observability assumptions, the effect of disturbance on regulated output may be made arbitrarily small in any finite bandwidth, but perfect rejection is obtained only at the steady state.

\subsection{Computational support with Matlab}

The algebraic operations concerning subspaces required to implement the algorithms of the geometric approach considered in this section are easily performed on the corresponding basis matrices by using some Matlab routines available in a diskette included with Basile and Marro (1992). Those directly related to the previously presented concepts are: 
$\mathrm{Q}=\operatorname{ima}(\mathrm{A}, \mathrm{p})$ Orthonormalization.

$Q=\operatorname{ortco}(A)$ Complementary orthogonalization.

$Q=\operatorname{sums}(A, B)$ Sum of subspaces.

$Q=\operatorname{ints}(A, B)$ Intersection of subspaces.

$Q=\operatorname{invt}(A, X)$ Inverse transform of a subspace.

$Q=\operatorname{ker}(A)$ Kernel of a matrix.

$\mathrm{Q}=\operatorname{mininv}(\mathrm{A}, \mathrm{B})$ Minimal $A$-invariant containing $\operatorname{im} B$.

$\mathrm{Q}=\operatorname{maxinv}(\mathrm{A}, \mathrm{X})$ Maximal $A$-invariant contained in $\operatorname{im} X$.

$[\mathrm{P}, \mathrm{Q}]=\operatorname{stabi}(\mathrm{A}, \mathrm{X})$ Matrices for the internal and external stability of the $A$-invariant $\operatorname{im} X$.

$\mathrm{Q}=\operatorname{mainco}(\mathrm{A}, \mathrm{B}, \mathrm{X})$ Maximal $(A, B)$-controlled invariant contained in $\operatorname{im} X$.

$\mathrm{Q}=\operatorname{minco}(\mathrm{A}, \mathrm{C}, \mathrm{X})$ Minimal $(A, C)$-conditioned invariant containing $\operatorname{im} X$.

$\mathrm{F}=\operatorname{effe}(\mathrm{A}, \mathrm{B}, \mathrm{X})$ State feedback matrix such that the $(A, B)$-controlled invariant $\operatorname{im} X$ is an $(A+B F)$-invariant.

$[\mathrm{P}, \mathrm{Q}]=\operatorname{stabv}(\mathrm{A}, \mathrm{B}, \mathrm{X}) \quad$ Matrices for the internal and external stabilizability of the $(A, B)$ controlled invariant $\operatorname{im} X$.

$\mathbf{z}=\operatorname{gazero}(\mathrm{A}, \mathrm{B}, \mathrm{C}, \mathrm{D})$ Invariant zeros of $(A, B, C)$ or $(A, B, C, D)$.

Let us complete the above computational machinery with the following $\mathrm{m}$-file, for constrained functional output controllability, hence useful to extend the concept of relative degree to the multivariable case.

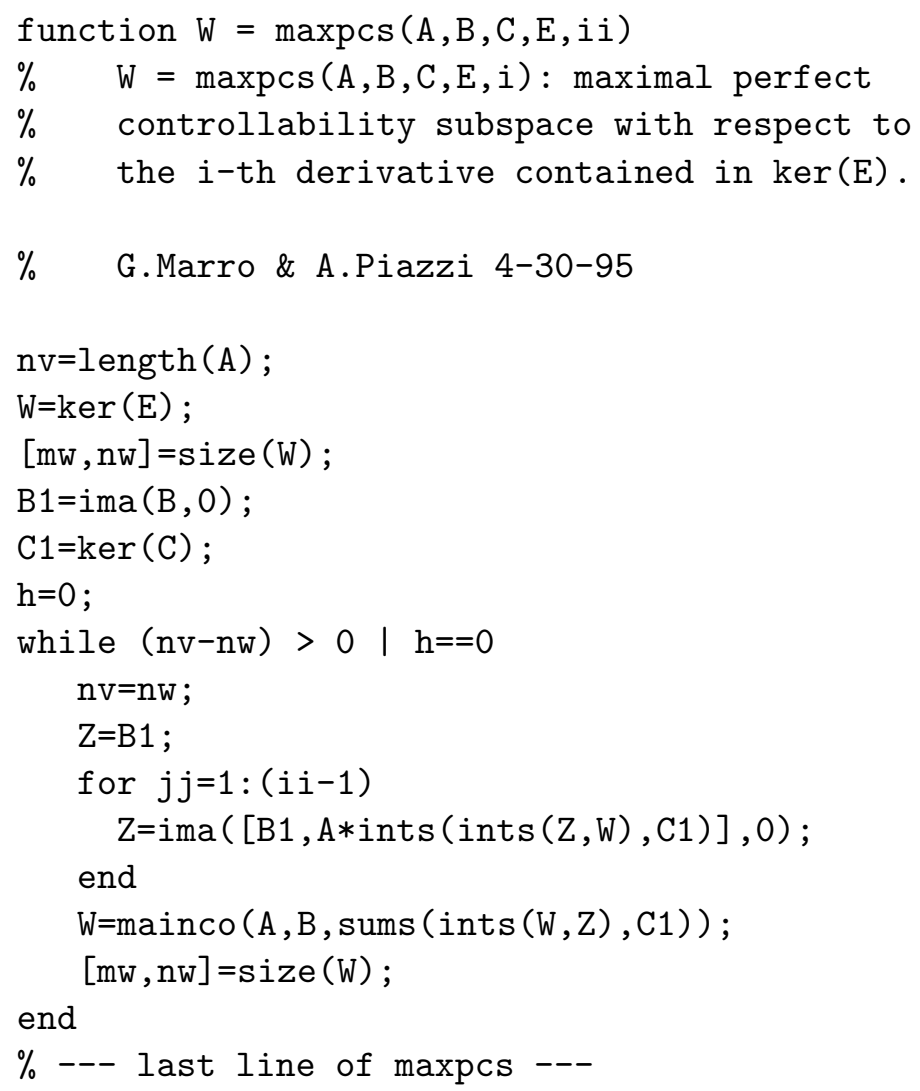

Notes and References. Most of the previously presented concepts and algorithms are reported 
in the books by Wonham [55] and Basile and Marro [11]. The geometric characterization of system invertibility and functional output controllability is due to Morse and Wonham [44], while constrained output controllability, hence the geometric-type extension of the relative degree concept to multivariable system, was introduced by Basile and Marro [8]. The elegant algorithm providing the reachable subspace on a controlled invariant by intersection with a conditioned invariant is due to Morse [43], and the role of invariant zeros in regulation problems was pointed out by Francis and Wonham [27]. The disturbance localization problem both with state and output feedback was presented by Basile and Marro as the first application of the geometric techniques in system synthesis [6], while the complete solution of this problem with output dynamic feedback and stability was given by Willems and Commault [53], and disturbance localization plus regulation by Schumacher [47]. Self-bounded controlled invariants were introduced by Basile, Marro and Piazzi $[9,12,13]$ to deal with stabilizability in a purely geometric context, and connection between invariant zeros and stabilizability in some wellknown synthesis problems, including disturbance localization, was investigated by Marro and Piazzi [38].

\section{Steady-State Robust Regulation with Feedback and the In- ternal Model}

This section extends some well-known properties of SISO feedback regulators to the MIMO case by using the previously introduced geometric techniques. The features of regulators considered here are: (i) closed-loop asymptotic stability or, more generally, pole assignability and (ii) the use of an internal model to achieve asymptotic tracking of the reference input and rejection of the disturbance input under the assumption that both reference and disturbance are generated by linear exosystems. Since the overall system considered, included the exosystems, is described by a linear homogeneous set of differential equations, whose initial state is the only variable affecting its evolution in time, we shall refer to this regulator design problem as the autonomous regulator problem. In order to make the comparison between the standard techniques based on transfer functions and the geometric techniques easier, a brief review of the SISO case is first presented.

\subsection{The SISO Autonomous Regulator Problem}

Let us refer to the closed-loop control system shown in Fig. 1, that includes a plant $\Sigma_{p}$, a regulator $\Sigma_{r}$ and two exosystems $\Sigma_{e 1}$ and $\Sigma_{e 2}$, generating the reference input $r$, to be tracked by the output $y$, and the disturbance input $d$, to be rejected at $y$, respectively. In this scheme the error $e$, whose zero setting detects efficiency of tracking and regulation, is the only input of the regulator, while the regulator output coincides with the manipulated input $u$ of the plant.

The transfer functions of the plant (given) and the regulator (to be determined) are

$$
G_{p}(s)=\frac{P_{p}(s)}{Q_{p}(s)}
$$

and

$$
G_{r}(s)=\frac{P_{r}(s)}{Q_{r}(s)}
$$




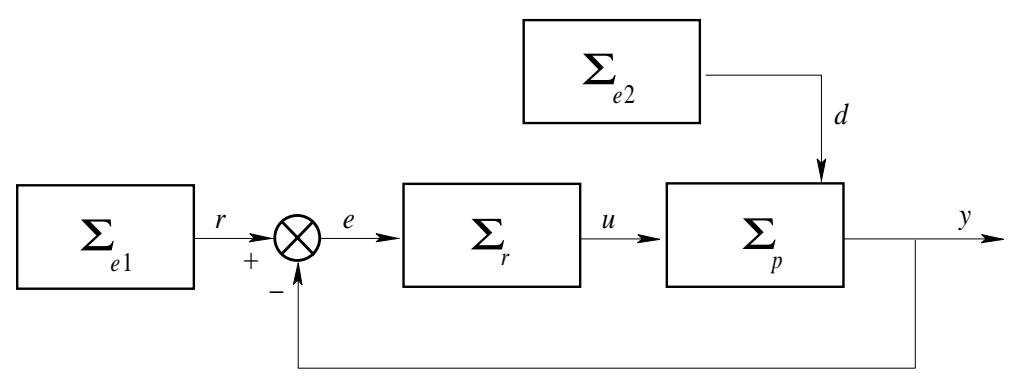

Figure 1: The standard closed-loop control system considered.

The polynomials $P_{p}(s)$, with degree $m_{p}$, and $Q_{p}(s)$, with degree $n_{p} \geq m_{p}$, are assumed to be relatively prime. The unknown polynomials $P_{r}(s)$, with degree $m_{r}$, and $Q_{r}(s)$, with degree $n_{r}$, must be such that $n_{r} \geq m_{r}$, in order to represent a causal system. The exosystems generate standard test signals, like steps, ramps, parabolas, sinusoids, possibly mixed. Let us suppose that the regulator is disconnected and denote by

$$
\frac{P_{e}(s)}{Q_{e}(s)}
$$

a strictly proper transfer function, with $P_{e}(s)$ having degree $n_{e}$, whose impulse response contains all the exosystem modes affecting $e$, that are assumed to be all not strictly stable. Clearly these modes are represented by the roots of $Q_{e}(s)$, thus all having non-negative real parts. To achieve asymptotic tracking and disturbance rejection it is necessary that the same modes are generated in the regulator and, possibly, in the plant, thus cancelling those of the exosystem at $e$. This artifice is called the internal model principle. Presence of the internal model and asymptotic stability of the regulation loop (with the exosystem disconnected) are the means to achieve asymptotic regulation and disturbance rejection.

The SISO autonomous regulator problem is: derive, if possible, a causal regulator $G_{r}(s)$ such that the closed-loop system of Fig. 1 is stable and $\lim _{t \rightarrow \infty} e(t)=0$ for all initial states of the exosystem, i.e., for all $P_{e}(s)$ in (3.2). In more formal mathematical terms it is stated as follows.

Problem 3.1 (The SISO Autonomous Regulator Problem) Given the transfer function $G_{p}(s)=P_{p}(s) / Q_{p}(s)$, and the polynomial $Q_{e}^{\prime}(s)$ such that $Q_{p}(s) Q_{e}^{\prime}(s)$ is divisible by $Q_{e}(s)$, find, if possible, polynomials $P_{r}(s)$ and $Q_{r}(s)$ defining a regulator such that

1. the degree of $P_{r}(s)$ is not greater than the degree of $Q_{r}(s)$;

2. $Q_{r}(s)$ is divisible by $Q_{e}^{\prime}(s)$;

3. all the roots of $Q_{c l}(s)=P_{r}(s) P_{p}(s)+Q_{r}(s) Q_{p}(s)$ are strictly stable.

Condition 1 expresses causality of the regulator, 2 the internal model principle and 3 strict stability of the control loop. The following theorem holds.

Theorem 3.1 (Solvability of the SISO Autonomous Regulator Problem) Problem 3.1 is solvable with $Q_{r}(s)$ having degree $n_{r} \geq n_{p}+n_{e}^{\prime}-1$ and all the roots of $Q_{c l}(s)$ arbitrary (hence, in particular, strictly stable) if and only if $Q_{e}(s)$ and $P_{p}(s)$ are relatively prime, i.e., if and only if all the zeros of the plant are different from the poles of the exosystem. ${ }^{7}$

\footnotetext{
${ }^{7}$ Augmenting the multiplicity of the internal model poles to compensate for a cancellation with zeros of the plant is not technically acceptable since produces unbounded $u$.
} 
A very straightforward proof of Theorem 3.1, well known in the literature, is based on the Diophantine equation

$$
A(s) X(s)+B(s) Y(s)=C(s)
$$

that is equivalent to a set of linear equations whose unknown quantities are the coefficients of $X(s)$ and $Y(s)$ and known parameters those of $A(s), B(s)$ and $C(s)$. The main properties of the Diophantine equation are recalled in the following statements.

Property 3.1 The Diophantine equation (3.4) is solvable with respect to $X(s)$ and $Y(s)$ if and only if $A(s)$ and $B(s)$ are relatively prime.

Property 3.2 Let us denote by $k$ and $h$ the degrees of $X(s)$ and $Y(s)$ respectively. The are related to the degrees $n, m$ and $l$ of $A(s), B(s)$ and $C(s)$ by the following relations:

$$
\begin{aligned}
& k= \begin{cases}l-n & \text { if } l>m+n \\
m & \text { if } l=m+n \\
m-1 & \text { if } l<m+n\end{cases} \\
& h=n-1
\end{aligned}
$$

Proof of Theorem 3.1. Let us consider the equation

$$
P_{r}(s) P_{p}(s)+Q_{e}^{\prime}(s) Q_{r}^{\prime}(s) Q_{p}(s)=Q_{c l}(s)
$$

whose member on the left is the characteristic polynomial of the closed-loop system considered. It is a Diophantine equation of the type (3.4) with $B(s):=P_{p}(s), A(s):=Q_{e}^{\prime}(s) Q_{p}(s)$, $C(s):=Q_{c l}(s)$. The plant being minimal and the assumption on the zeros of the plant ensure the condition stated in Property 3.1. Let $Q_{c l}(s)$ be a polynomial having degree $l=2 n_{p}+n_{e}^{\prime}-1$ with arbitrary roots. In this case the degrees of $Y(s)=P_{r}(s)$ and $X(s)=Q_{r}^{\prime}(s)$ are $m=m_{p}$, $n=n_{p}+n_{e}^{\prime}$, so that relations (3.5) and (3.6) give $n_{r}^{\prime}=n_{p}-1$, hence $n_{r}=n_{p}+n_{e}^{\prime}-1$, and $m_{r}=n_{r}$. A greater value of $l$ simply produces $n_{r}$ to be increased by the same amount. ${ }^{8}$

It is worth noting that the internal model produces zeros equal to the exosystem poles in the transfer functions from $r$ to $e$ and from $d$ to $e$, thus making asymptotic zero setting of $e$ possible. In fact, let us assume $Q_{r}(s)=Q_{e}^{\prime}(s) Q_{r}^{\prime}(s)$. This follows from

$$
\frac{E(s)}{R(s)}=\frac{1}{1+G_{r}(s) G_{p}(s)}=\frac{Q_{e}^{\prime}(s) Q_{r}^{\prime}(s) Q_{p}(s)}{P_{r}(s) P_{p}(s)+Q_{e}^{\prime}(s) Q_{r}^{\prime}(s) Q_{p}(s)}
$$

since $Q_{e}(s)$ is a divisor of $Q_{e}^{\prime}(s) Q_{p}(s)$ by assumption.

Algorithm 3.1 (Solution of the Diophantine Equation) Although the solution of the Diophantine equation is treated in many textbooks, a very concise outline of it, consistent with the previous arguments and symbols, is repeated here for the reader's convenience. Polynomial $A(s)$

\footnotetext{
${ }^{8}$ A more general result, directly obtainable from the Diophantine equation, is stated as follows: it is possible to derive a regulator with $n_{f}$ fixed poles and $m_{f}$ fixed zeros while preserving assignability of all the closedloop poles; it has the minimal order $n_{r}=m_{r}=n_{p}+n_{f}+m_{f}-1$, while the number of closed-loop poles is $l=2 n_{p}+n_{f}+m_{f}-1$.
} 
is assumed to be monic (if not, divide the leading coefficient of polynomials $A(s), B(s)$ and $C(s)$ by $\left.a_{n}\right)$. Let

$$
\begin{aligned}
& A(s):=s^{n}+a_{n-1} s^{n-1}+\ldots+a_{0} \\
& B(s):=b_{m} s^{m}+b_{m-1} s^{m-1}+\ldots+b_{0} \\
& C(s):=c_{l} s^{l}+c_{l-1} s^{l-1}+\ldots+c_{0} \\
& X(s):=x_{k} s^{k}+x_{k-1} s^{k-1}+\ldots+x_{0} \\
& Y(s):=y_{h} s^{h}+y_{h-1} s^{h-1}+\ldots+y_{0}
\end{aligned}
$$

be the polynomials considered, with $A(s)$ and $B(s)$ satisfying the condition stated in Property 3.1. To maintain notation within acceptably simple terms we refer to the particular case $n=3, m=2$, $l=8$, hence $h=2, k=5$. In this particular case the set of linear equations equivalent to (3.4) appears in the form

$$
x_{5}=c_{8}, \quad\left[\begin{array}{cccccccc}
1 & 0 & 0 & 0 & 0 & 0 & 0 & 0 \\
a_{2} & 1 & 0 & 0 & 0 & 0 & 0 & 0 \\
a_{1} & a_{2} & 1 & 0 & 0 & 0 & 0 & 0 \\
a_{0} & a_{1} & a_{2} & 1 & 0 & b_{2} & 0 & 0 \\
0 & a_{0} & a_{1} & a_{2} & 1 & b_{1} & b_{2} & 0 \\
0 & 0 & a_{0} & a_{1} & a_{2} & b_{0} & b_{1} & b_{2} \\
0 & 0 & 0 & a_{0} & a_{1} & 0 & b_{0} & b_{1} \\
0 & 0 & 0 & 0 & a_{0} & 0 & 0 & b_{0}
\end{array}\right]\left[\begin{array}{c}
x_{4} \\
x_{3} \\
x_{2} \\
x_{1} \\
x_{0} \\
y_{2} \\
y_{1} \\
y_{0}
\end{array}\right]=\left[\begin{array}{c}
c_{7}-c_{8} a_{2} \\
c_{6}-c_{8} a_{1} \\
c_{5}-c_{8} a_{0} \\
c_{4} \\
c_{3} \\
c_{2} \\
c_{1} \\
c_{0}
\end{array}\right]
$$

If $l \geq m+n$ like in the above example, the system matrix is $l \times l$, with the first $k$ columns reporting the coefficients of $A(s)$ in a band as shown and the last $l-k$ columns those of $B(s)$ in a band justified to foot; in the first $n$ elements of the column matrix at the right the coefficients of $A(s)$ from the second up, each multiplied by the first coefficient of $C(s)$, are subtracted from the coefficients of $C(s)$ from the second up as shown. If $l<m+n$, the system matrix is $(m+n) \times(m+n)$ and built in the same way, while the leading coefficients of $C(s)$ appearing in (3.14) are set equal to zero.

\subsection{Robustness of regulation in the SISO case}

The asymptotic tracking and disturbance rejection property obtained by using a regulator including an accurate and robust replica of the exosystem eigenstructure has the remarkable property of being robust with respect to plant parameter variations so long as closed-loop stability is maintained. The reason for this can be clearly pointed out by analyzing the observability property of a state space realization of the SISO system considered in the previous subsection. Let $\left(A_{1}, B_{1}, C_{1}\right),(N, M, L, K)$ be the state space realizations of the plant and the regulator, $A_{2}$ be a matrix having the structure of the overall exosystem, i.e., reproducing all the modes of the exosystem $\Sigma_{e 1}$ and $\Sigma_{e 2}$ observable at $e$ with the regulator disconnected. Referring again to Fig. 1, we use for the regulated system (the plant plus the exosystems) the following state space representation

$$
\begin{aligned}
\dot{x}(t) & =A x(t)+B u(t) \\
e(t) & =E x(t)
\end{aligned}
$$


with

$$
x:=\left[\begin{array}{l}
x_{1} \\
x_{2}
\end{array}\right], \quad A:=\left[\begin{array}{cc}
A_{1} & A_{3} \\
O & A_{2}
\end{array}\right], \quad B:=\left[\begin{array}{c}
B_{1} \\
O
\end{array}\right], \quad E:=\left[\begin{array}{ll}
E_{1} & E_{2}
\end{array}\right]
$$

Matrices $A_{1}, B_{1}$ and $C_{1}$ are assumed to be $n_{1} \times n_{1}, n_{1} \times 1$ and $1 \times n_{1}$ respectively, while $A_{2}$, that models the exosystem eigenstructure, is assumed to be $n_{2} \times n_{2} . A_{3}, n_{1} \times n_{2}$, represents the influence of the exosystem $\Sigma_{e 2}$ on the plant, while $E_{1}$ and $E_{2}$ are set equal to $-C_{1}$ and $C_{2}$ respectively, where $C_{2}, 1 \times n_{2}$, is the output matrix of $A_{2}$ producing the reference $r$. The regulator equations are

$$
\begin{aligned}
\dot{z}(t) & =N z(t)+M e(t) \\
u(t) & =L z(t)+K e(t)
\end{aligned}
$$

where $N, M, L$ are $m \times m, m \times 1$ and $1 \times m$ respectively, while $K$ is a scalar. Consistency with the previous transfer function description implies $n=n_{p}, m=n_{r}$ and $n_{2}=n_{e}$. Fig. 2, a shows
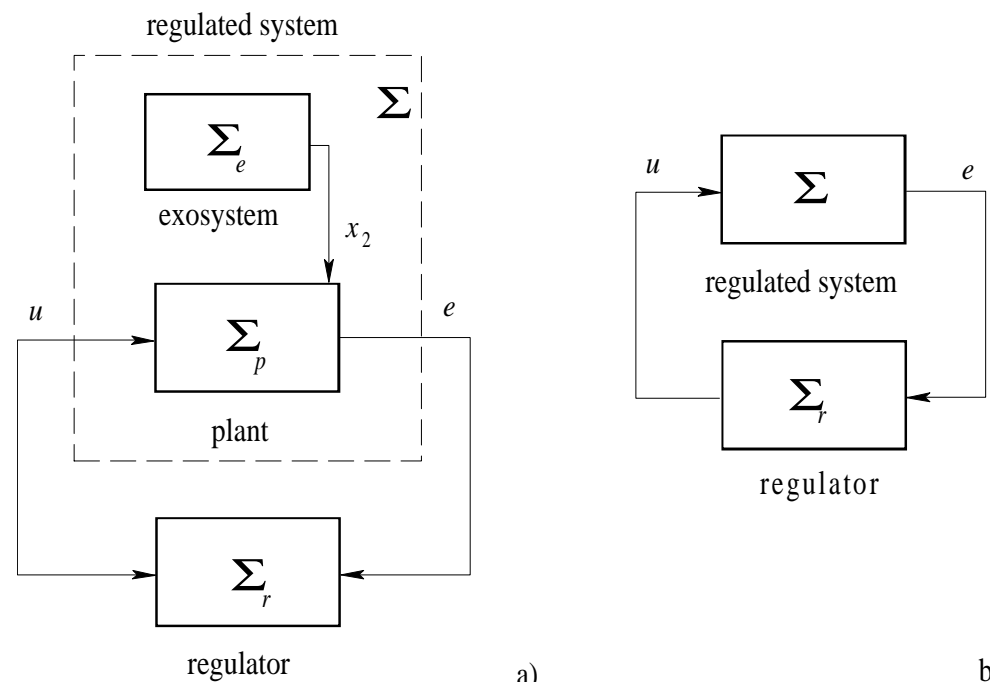

Figure 2: Block diagrams referring to the state-space representation of a MIMO system.

the standard connection of the exosystem, plant and regulator, while Fig. 2,b points out the essence of the autonomous regulator problem: the regulated system $\Sigma$ (given) with its closedloop connection with the regulator $\Sigma_{r}$ (to be determined). The overall system is described as the autonomous extended system

$$
\begin{aligned}
\dot{\hat{x}}(t) & =\hat{A} \hat{x}(t) \\
e(t) & =\hat{E} \hat{x}(t)
\end{aligned}
$$

with

$$
\begin{aligned}
\hat{A} & :=\left[\begin{array}{ccc}
A_{1}+B_{1} K E_{1} & A_{3}+B_{1} K E_{2} & B_{1} L \\
O & A_{2} & O \\
M E_{1} & M E_{2} & N
\end{array}\right], \hat{x}:=\left[\begin{array}{c}
x_{1} \\
x_{2} \\
z
\end{array}\right], \\
\hat{E} & :=\left[\begin{array}{lll}
E_{1} & E_{2} & O
\end{array}\right]
\end{aligned}
$$

Referring to (3.18), the asymptotic tracking and regulation property is explained in geometric terms as follows: assume that the pair $(E, A)$ is observable; this implies, in particular, that the 
exosystem modes may all affect $e$. If a regulator with the internal model is connected as shown, thus replicating the same modes at $e$ (cancellation with zeros of the plant is not possible by assumption) these become unobservable since for any initial state of the exosystem there is a suitable initial state of the overall system and the internal model that cancels the corresponding modes at $e$; hence, the autonomous system (3.18) has the unobservability subspace containing these modes, that are all not strictly stable by assumption. In other words, an $\hat{A}$-invariant $\hat{\mathcal{W}} \subseteq \operatorname{ker} \hat{E}$ having dimension $n_{2}$ exists, that is internally not strictly stable. Since the eigenvalues of $\hat{A}$ are clearly those of $A_{2}$ plus those of the regulation loop, that are strictly stable, $\hat{\mathcal{W}}$ is externally strictly stable. Hence $\left.\hat{A}\right|_{\hat{\mathcal{W}}}$ has the eigenstructure of $A_{2}\left(n_{2}\right.$ eigenvalues) and $\left.\hat{A}\right|_{\hat{\mathcal{X}}} / \hat{\mathcal{W}}$ that of the control loop $\left(n_{1}+m\right.$ eigenvalues).

If the internal model is maintained, unobservability of the exosystem corresponding modes is also maintained in presence of plant parameter changes. In geometric terms, the existence of the $\hat{A}$-invariant $\hat{\mathcal{W}} \subseteq \operatorname{ker} \hat{E}$ is preserved under parameter changes.

\section{Remarks:}

1. Robustness of the internal model is easily achievable when its eigenvalues are all zero, i.e., when the signals to be asymptotically tracked or rejected are linear combinations of steps, ramps, parabolas, etc.

2. Since the closed-loop poles are all assignable, the modes of the error variable are completely arbitrary.

3. The invariant zeros of $(A, B, E)$ are those of the plant $\left(A_{1}, B_{1}, C_{1}\right)$ plus the eigenvalues of $A_{2}$.

4. It is possible that the poles of the overall exosystem are a subset of those of the internal model (for instance, a double pole at the origin in the internal model produces steady-state regulation also with an exosystem having a single pole at the origin).

\subsection{The MIMO Autonomous Regulator Problem}

Let us consider again equations (3.15)-(3.17) and refer them to the MIMO case, by assuming that matrices $B_{1}$ and $C_{1}$ are now $n_{1} \times p$ and $q \times n_{1}$, while $M, L, K$ and $C_{2}$ are $m \times q, p \times m$, $p \times q$ and $q \times n_{2}$ respectively. We also assume that $\left(A_{1}, B_{1}\right)$ is reachable and $(E, A)$ observable. ${ }^{9}$

In the corresponding state space $\mathcal{X}$ with dimension $n:=n_{1}+n_{2}$ we define the subspace plant $\mathcal{P}$ through

$$
\mathcal{P}:=\left\{x: x_{2}=0\right\}=\operatorname{im}\left[\begin{array}{c}
I_{n_{1}} \\
O
\end{array}\right]
$$

Similarly, in the extended state space $\hat{\mathcal{X}}$ with dimension $\hat{n}:=n_{1}+n_{2}+m$ the extended plant $\hat{\mathcal{P}}$ is defined as

$$
\hat{\mathcal{P}}:=\left\{\hat{x}: x_{2}=0\right\}=\operatorname{im}\left[\begin{array}{cc}
I_{n_{1}} & O \\
O & O \\
O & I_{m}
\end{array}\right]
$$

\footnotetext{
${ }^{9}$ Relaxing these assumptions to $\left(A_{1}, B_{1}\right)$ being stabilizable and $(E, A)$ detectable does not significantly affect the solution: the only consequence is that the error dynamics is no longer arbitrary.
} 
Clearly $\mathcal{P}$ and $\hat{\mathcal{P}}$ are an $A$-invariant and an $\hat{A}$-invariant respectively.

The MIMO autonomous regulator problem is: derive, if possible, a regulator $(N, M, L, K)$ such that the closed-loop system with the exosystem disconnected is stable and $\lim _{t \rightarrow \infty} e(t)=0$ for all initial states of the autonomous extended system. In geometric terms it is stated as follows.

Problem 3.2 (The MIMO Autonomous Regulator Problem) Let us refer to the extended system (3.18) and assume $\hat{\mathcal{E}}:=\operatorname{ker} \hat{E}$. Given the mathematical model (3.15)-(3.16) of the plant and the exosystem determine, if possible, a regulator $(N, M, L, K)$ such that $\hat{\mathcal{P}}$ is internally stable and an $\hat{A}$-invariant $\hat{\mathcal{L}}$ exists satisfying

1. $\hat{\mathcal{L}} \subseteq \hat{\mathcal{E}}$

2. $\sigma\left(\left.\hat{A}\right|_{\hat{\mathcal{X}} / \hat{\mathcal{L}}}\right) \subseteq \mathbf{C}_{-}$.

Lemma 3.1 Problem 3.2 has a solution if and only if an $\hat{A}$-invariant $\hat{\mathcal{W}}$ exists such that

$$
\begin{aligned}
& \hat{\mathcal{W}} \subseteq \hat{\mathcal{E}} \\
& \hat{\mathcal{W}} \oplus \hat{\mathcal{P}}=\hat{\mathcal{X}} \\
& \sigma\left(\left.\hat{A}\right|_{\hat{\mathcal{X}} / \hat{\mathcal{W}}}\right) \subseteq \mathbf{C}_{-}
\end{aligned}
$$

Proof. Only if. Suppose that Problem 3.2 has a solution and let $\hat{\mathcal{W}}^{+}$be the subspace of the nonstrictly stable modes of $\hat{A}$, that is an $\hat{A}$-invariant. Since the only non-strictly stable eigenvalues are those of $A_{2}$, while the other eigenvalues, strictly stable, are those of the regulation loop, i.e., those of $\left.\hat{A}\right|_{\hat{\mathcal{P}}}$, the dimension of $\hat{\mathcal{W}}^{+}$is $n_{2}$. Hence, by the requirement 2 of Problem 3.2, $\hat{\mathcal{W}}^{+} \subseteq \hat{\mathcal{L}}$. It follows that $\hat{\mathcal{W}}^{+}$is the minimum $\hat{A}$-invariant meeting the geometric conditions of Problem 3.2.

If. Assume $\hat{\mathcal{L}}:=\hat{\mathcal{W}}^{+}$in the statement of Problem 3.2.

Owing to (3.23) $\hat{\mathcal{W}}$ can be expressed as the image of a basis matrix with a particular structure, i.e.,

$$
\hat{\mathcal{W}}=\operatorname{im} \hat{W} \quad \text { with } \hat{W}=\left[\begin{array}{c}
X_{1} \\
I_{n_{2}} \\
Z
\end{array}\right]
$$

The main Theorem on asymptotic regulation in the MIMO case is stated in strictly geometric terms as follows.

Theorem 3.2 (Solvability of the MIMO Autonomous Regulator Problem: non-constructive necessary and sufficient conditions) Let us assume $\mathcal{E}:=\operatorname{ker} E$. Problem 3.2 admits a solution if and only if an $(A, \mathcal{B})$-controlled invariant $\mathcal{V}$ exists such that

$$
\begin{aligned}
& \mathcal{V} \subseteq \mathcal{E} \\
& \mathcal{V} \oplus \mathcal{P}=\mathcal{X}
\end{aligned}
$$

Proof. Only if. Let us refer to (3.25), with $\hat{\mathcal{W}}$ satisfying the properties stated in Lemma 3.1 and assume

$$
\mathcal{V}=\operatorname{im} V \text { with } V=\left[\begin{array}{l}
X_{1} \\
I_{n_{2}}
\end{array}\right]
$$


$\mathcal{V}$ is an $(A, \mathrm{im} B)$-controlled invariant. In fact, owing to (2.4) a matrix $S$ exists such that $\hat{A} \hat{W}=\hat{W} S$, or

$$
\left[\begin{array}{c}
\left(A_{1}+B_{1} K E_{1}\right) X_{1}+A_{3}+B_{1} K E_{2}+B_{1} L Z \\
A_{2} \\
M E_{1} X_{1}+M E_{2}+N Z
\end{array}\right]=\left[\begin{array}{c}
X_{1} \\
I_{n_{2}} \\
Z
\end{array}\right] S
$$

By suitably collecting elements we obtain

$$
\left[\begin{array}{c}
A_{1} X_{1}+A_{3} \\
A_{2}
\end{array}\right]=\left[\begin{array}{c}
X_{1} S-B_{1}\left(K E_{1} X_{1}+K E_{2}+L Z\right) \\
S
\end{array}\right]
$$

that can be expressed as $A V=V S+B U$, so that $\mathcal{V}$ is an $(A, \mathcal{B})$-controlled invariant by virtue of (2.13). Due to the particular structure of $\hat{E}$ shown in (3.19), from $\hat{E} \hat{W}=O$ it follows that $E V=O$, hence (3.26) holds. Finally, (3.27) immediately follows from the structure of the basis matrix $V$.

If. Let $\mathcal{V}$ be an $(A, \mathcal{B})$-controlled invariant satisfying (3.27), hence having a basis matrix $V$ with the structure shown in (3.28): there exists a state-feedback matrix $F=\left[F_{1} F_{2}\right]$, partitioned according to (3.16), such that $(A+B F) \mathcal{V} \subseteq \mathcal{V}$ and the eigenvalues of $A_{1}+B_{1} F_{1}$ are arbitrarily assigned. In fact, consider the similarity transformation defined as $T=\left[T_{1} T_{2}\right]$ with $T_{1}=\left[I_{n_{1}} O\right]^{T}$, $T_{2}=V$. The transformed matrices $A^{\prime}:=T^{-1} A T, B^{\prime}:=T^{-1} B$, and $E^{\prime}:=E T$ have the structures

$$
A^{\prime}=\left[\begin{array}{cc}
A_{1} & A_{3}^{\prime} \\
O & A_{2}
\end{array}\right], \quad B^{\prime}=\left[\begin{array}{c}
B_{1} \\
O
\end{array}\right], \quad E^{\prime}=\left[\begin{array}{ll}
E_{1} & O
\end{array}\right]
$$

Since $\left(A_{1}, B_{1}\right)$ is reachable, a matrix $F_{1}$ exists such that $A_{1}+B_{1} F_{1}$ has any given set of $n_{1}$ eigenvalues; since $\mathcal{V}$ is a controlled invariant, a matrix $F_{2}^{\prime}$ exists such that $A_{3}^{\prime}+B_{1} F_{2}^{\prime}=O$. $F$ referred to the original basis is computed as $F=\left[\begin{array}{ll}F_{1} & F_{2}^{\prime}\end{array}\right] T^{-1}$. Furthermore, let $G$ be such that $A+G E$ has any given set of $n$ eigenvalues, which is possible since $(E, A)$ is observable. We claim that the regulator defined by

$$
N:=A+B F+G E, \quad M:=-G, \quad L:=F, \quad K:=O
$$

solves Problem 3.2. In fact Lemma 3.1 is satisfied with

$$
\hat{A}=\left[\begin{array}{cc}
A & B F \\
-G E & A+B F+G E
\end{array}\right], \quad \hat{E}=\left[\begin{array}{cc}
E & O
\end{array}\right], \quad \hat{W}=\left[\begin{array}{c}
V \\
V
\end{array}\right]
$$

as is shown with the similarity transformation defined by

$$
T=T^{-1}=\left[\begin{array}{cc}
I_{n} & O \\
I_{n} & -I_{n}
\end{array}\right]
$$

The corresponding matrices $\hat{A}^{\prime}:=T^{-1} \hat{A} T, \hat{E}^{\prime}:=\hat{E} T$ and $\hat{W}^{\prime}:=T^{-1} \hat{W}$ appear in the forms

$$
\hat{A}^{\prime}=\left[\begin{array}{cc}
A+B F & -B F \\
O & A+G E
\end{array}\right], \quad \hat{E}^{\prime}=\left[\begin{array}{ll}
E & O
\end{array}\right], \quad \hat{W}^{\prime}=\left[\begin{array}{c}
V \\
O
\end{array}\right]
$$


that allow easy checking of conditions stated in Lemma 3.1, by also using the previously defined partitioning of the involved submatrices.

Conditions stated in Theorem 3.2, although necessary and sufficient, are non-constructive, since the existence of a controlled invariant satisfying (3.26), (3.27), that is here called the resolvent, is not easy to check. However, the "if" part of the proof provides an algorithm to derive a controller with the internal model of the exosystem when the resolvent is given. The order of the controller is $n=n_{1}+n_{2}$ (that of the plant plus that of the exosystem) like in the SISO case. The following theorem gives conditions similar to those of Theorem 3.1 for the SISO case that are also only sufficient, but very important in practice, since avoid unboundedness of the manipulated input $u$ due to pole-zero cancellation between the plant and the regulator.

Theorem 3.3 (Solvability of the MIMO Autonomous Regulator Problem: constructive sufficient and almost necessary ${ }^{10}$ conditions). Let us consider $\mathcal{V}^{*}:=\max \mathcal{V}(A, \mathcal{B}, \mathcal{E})$. Problem 3.2 admits a solution if

$$
\begin{aligned}
& \mathcal{V}^{*}+\mathcal{P}=\mathcal{X} \\
& \mathcal{Z}\left(A_{1}, B_{1}, E_{1}\right) \cap \sigma\left(A_{2}\right)=\emptyset
\end{aligned}
$$

Proof. We show that the stated conditions allow computation of a resolvent. Let $F$ be a matrix such that $(A+B F) \mathcal{V}^{*} \subseteq \mathcal{V}^{*}$ and introduce the similarity transformation $T:=\left[\begin{array}{lll}T_{1} & T_{2} & T_{3}\end{array}\right]$, with $\operatorname{im} T_{1}=\mathcal{V}^{*} \cap \mathcal{P}, \operatorname{im}\left[T_{1} T_{2}\right]=\mathcal{V}^{*}$ and $\operatorname{im}\left[T_{1} T_{3}\right]=\mathcal{P}$. Recall that $\mathcal{P}$ is an $A$-invariant and note that, owing to the particular structure of $B$, it also is an $(A+B F)$-invariant for any $F$. In the new basis the linear transformation $A+B F$ has the structure

$$
A^{\prime}=T^{-1}(A+B F) T=\left[\begin{array}{ccc}
A_{11}^{\prime} & A_{12}^{\prime} & A_{13}^{\prime} \\
O & A_{22}^{\prime} & O \\
O & O & A_{33}^{\prime}
\end{array}\right]
$$

By a dimensionality argument the eigenvalues of the exosystem are those of $A_{22}^{\prime}$, while the invariant zeros of $\left(A_{1}, B_{1}, E_{1}\right)$ are a subset of $\sigma\left(A_{11}^{\prime}\right)$ since $\mathcal{R}_{\mathcal{V}^{*}}$ is contained in $\mathcal{V}^{*} \cap \mathcal{P}$. All the other elements of $\sigma\left(A_{11}^{\prime}\right)$ are arbitrarily assignable with $F$. Hence, owing to (3.37), the Sylvester equation

$$
A_{11}^{\prime} X-X A_{22}^{\prime}=-A_{12}^{\prime}
$$

admits a unique solution. The matrix $V:=T_{1} X+T_{2}$ is a basis matrix of an $(A, \mathcal{B})$-controlled invariant $\mathcal{V}$ satisfying (3.26) and (3.27).

If $\mathcal{V}^{*} \cap \mathcal{B}=\{0\}$, matrix $A_{11}^{\prime}$ is independent of $F$. Thus the following corollary is also proved.

Corollary 3.1 (Uniqueness of the resolvent) If the plant is invertible and (3.36), (3.37) are satisfied, a unique $(A, \mathcal{B})$-controlled invariant $\mathcal{V}$ satisfying conditions (3.26), (3.27) exists.

\section{Remarks:}

1. The proof of Theorem 3.3 provides the computational framework to derive a resolvent when the sufficient conditions stated (that are also necessary if the input of the plant must remain bounded) are satisfied.

\footnotetext{
${ }^{10}$ The conditions are necessary if the control variable $u$ must remain bounded: like in the SISO case, this is possible also when the output $y$ is unbounded if a part of the internal model is contained in the plant.
} 
2. Relations (3.36) and (3.37) are respectively a structural condition and a spectral condition; they are easily checkable by means of Algorithms 2.6-2.8 described in the previous section.

3. When a resolvent has been determined by means of the computational procedure described in the proof of Theorem 3.3, it can be used to derive a regulator with the procedure outlined in the "if" part of the proof of Theorem 3.2.

4. The order of the obtained regulator is $n$ (that of the plant plus that of the exosystem) with the corresponding $2 n_{1}+n_{2}$ closed-loop eigenvalues completely assignable under the assumption that $\left(A_{1}, B_{1}\right)$ is reachable and $(E, A)$ observable.

5. The internal model principle is satisfied since the eigenstructure of the regulator system matrix $(A+B F+G E)$ contains that of $A_{2}$ : in fact the subspace $\mathcal{V}$, that is an $(A+B F)$ invariant with the internal eigenstructure equal to that of $A_{2}$, is also an $(A+B F+G E)$ invariant with the same internal eigenstructure because it is contained in ker $E$. Thus, if we consider the connection from the exosystem to the overall system as the input of the feedback loop and the error variable $e$ as the output, the eigenstructure of $A_{2}$ is a part of the invariant zero structure.

\subsection{Robustness of regulation in the MIMO case}

Unfortunately, having the eigenstructure of the exosystem as a part of the invariant zero structure of the feedback loop is not sufficient to obtain robustness of regulation in presence of small parameter changes also in the MIMO case. The reason for this can be explained in simple terms as follows: an invariant zero of a generic triple $(A, B, C)$ is, according to Definition 2.9 , an internal unassignable eigenvalue of the maximal $(A, \mathcal{B})$ controlled invariant contained in $\mathcal{C}$ and if the corresponding mode is suitably injected at the input, an initial state nulling the output exists. In the multivariable regulation loop derived in the proof of Theorem 3.2 the internal model actually nulls the modes of the exosystem appearing at $e$, since produces corresponding zeros in the closed-loop system, but only if they are injected in the loop in the precise way represented by the mathematical model $(A, B, E)$ assumed: if this is subject to parameter variations, since the influence of each mode on each output is subject to change unpredictibly and independently of those of the corresponding mode generated in the regulator, asymptotic perfect zero setting of the error is no longer possible.

A possible remedy for this is to repeat the exosystem internal model in the regulator once for each controlled output, thus making automatic compensation of any parameter drift possible, if it is small enough to preserve the strict stability of the regulation loop. We shall show with a constructive argument that such an asymptotically robust regulator exists if the conditions stated in Theorem 3.2 are satisfied, without any additional requirement.

Algorithm 3.2 (The Francis Multivariable Asymptotically Robust Regulator) Assume as the matrix of the internal model

$$
A_{2 e}:=\left[\begin{array}{cccc}
A_{2} & O & \ldots & O \\
O & A_{2} & \ldots & O \\
\vdots & \vdots & \ddots & \vdots \\
O & O & \ldots & A_{2}
\end{array}\right]
$$


where $A_{2}$ is replicated as many times as there are regulated output components. Let $n_{2 e}$ be the dimension of $A_{2 e}$. Then define the extension of the controlled system

$$
A_{e}:=\left[\begin{array}{cc}
A_{1} & A_{3 e} \\
O & A_{2 e}
\end{array}\right], \quad B_{e}:=\left[\begin{array}{c}
B_{1} \\
O
\end{array}\right], \quad E_{e}:=\left[\begin{array}{ll}
E_{1} & O
\end{array}\right], \quad P_{e}:=\left[\begin{array}{c}
I_{n_{1}} \\
O
\end{array}\right]
$$

where $A_{3 e}$ has been determined under the only requirement that $\left(E_{e}, A_{e}\right)$ is observable. The existence of any $(A, \mathcal{B})$-controlled invariant $\mathcal{V} \subseteq \mathcal{E}$ satisfying $\mathcal{V}+\mathcal{P}=\mathcal{X}$ implies that $B_{1}$ is such that every mode of the exosystem appearing at $e$ (from which the exosystem is completely observable) can be cancelled by a suitable feedback from the state of the exosystem itself to $u$. Since this is an intrinsic property of $\left(A_{1}, B_{1}, E_{1}\right)$, it is also valid for the new system (3.41) i.e., $\mathcal{V}^{*}+\mathcal{P}=\mathcal{X}$ implies $\mathcal{V}_{e}^{*}+\mathcal{P}_{e}=\mathcal{X}_{e}$ with $\mathcal{V}_{e}^{*}:=\max \mathcal{V}\left(A_{e}, \mathcal{B}_{e}, \mathcal{E}_{e}\right)\left(\mathcal{E}_{e}:=\operatorname{ker} E_{e}\right)$. Since the conditions of Theorem 3.3 are also valid for $\mathcal{V}_{e}^{*}$, an $\left(A_{e}, \mathcal{B}_{e}\right)$-controlled invariant $\mathcal{V}_{e}$ exists such that $\mathcal{V}_{e} \oplus \mathcal{P}_{e}=\mathcal{X}_{e}$. Determine $F_{e}=\left[\begin{array}{ll}F_{1} & F_{2 e}\end{array}\right]$ such that $\left(A_{e}+B_{e} F_{e}\right) \mathcal{V}_{e} \subseteq \mathcal{V}_{e}$ and $A_{1}+B_{1} F_{1}$ is stable, $G_{e}$ such that $A_{e}+G_{e} E_{e}$ is stable, and assume

$$
N:=A_{e}+B_{e} F_{e}+G_{e} E_{e}, \quad M:=-G_{e}, \quad L:=F_{e}, \quad K:=O
$$

With this assumption the conditions stated in Lemma 3.1 hold. In fact, from

$$
\hat{A}=\left[\begin{array}{ccc}
A_{1} & A_{3} & B_{1} F_{e} \\
O & A_{2} & O \\
-G_{e} E_{1} & -G_{e} E_{2} & A_{e}+B_{e} F_{e}+G_{e} E_{e}
\end{array}\right], \quad \hat{E}=\left[\begin{array}{lll}
E_{1} & E_{2} & O
\end{array}\right]
$$

by means of the similarity transformation

$$
T=T^{-1}:=\left[\begin{array}{ccc}
I_{n_{1}} & O & O \\
O & I_{n_{2}} & O \\
R & O & -I_{n_{e}}
\end{array}\right], \text { with } R:=\left[\begin{array}{c}
I_{n_{1}} \\
O
\end{array}\right]
$$

we derive as $\hat{A}^{\prime}:=T^{-1} \hat{A} T$ and $\hat{E}^{\prime}:=\hat{E} T$ the matrices

$$
\hat{A}^{\prime}=\left[\begin{array}{ccc}
A_{1}+B_{1} F_{1} & A_{3} & -B_{1} F_{e} \\
O & A_{2} & O \\
O & S & A+G_{e} E_{e}
\end{array}\right], \quad \hat{E}^{\prime}=\left[\begin{array}{lll}
E_{1} & E_{2} & O
\end{array}\right]
$$

In (3.44) $n_{e}:=n_{1}+n_{2 e}$ and $R$ is assumed to be $n_{e} \times n_{1}$ while in (3.45) $S$ denotes a nonzero matrix. Since the closed-loop system is obtained from (3.45) by deleting the second row and column, we conclude that the eigenvalues of the regulation loop can be arbitrarily assigned. Owing to the presence of a "robust" internal model, that sets asymptotically to zero all the exosystem modes at every component of $e$, the pair $(\hat{E}, \hat{A})$ has a "robust" unobservability subspace $\hat{\mathcal{W}}$ with dimension $n_{2}$ that can be computed both as $\max \mathcal{L}(\hat{A}, \hat{E})$ or as the unique complement of the $\hat{A}$-invariant $\hat{\mathcal{P}}$.

\section{Remarks:}

1. Algorithm 3.2 allows achievement of asymptotic robustness with a regulator of order $n_{1}+q \times n_{2 e}, q$ being the number of the regulated output components (the components of $e$, assumed to be as many as the components of the output $y$ of the plant). 
2. The order of the regulator can be reduced if for each regulated output $e_{i}$ an internal model is used, reproducing only the maximal eigenstructure of the exosystem observable at $e_{i}$ when parameters change. This can be obtained by choosing suitable submatrices in $A_{2 e}$ and $A_{3 e}$ that, for every eigenvalue of the exosystem, make observable at $e_{i}$ an eigenstructure belonging to the internal model and the plant equal to the eigenstructure of the exosystem that is observable at $e_{i}$.

3. The plant can complete the action of a particular internal model, thus making reduction of its order possible, if some of its eigenvalues are equal to those of the exosystem observable at the corresponding output; however, these eigenvalues of the plant must be suitably shared between outputs, avoiding repetitions that may cause lack of robustness of regulation.

Notes and References. In many textbooks on automatic control systems pole assignment is solved, even in the SISO case, by converting the system transfer function into a quadruple $(A, B, C, D)$ and using state feedback through an full-order or a reduced-order observer. The reason for this is probably only a historical habit, since resort to the Diophantine equation appears to be more convenient, at least in the SISO case, since it gives the result directly in polynomial form. It also makes it possible to introduce any given transfer function as a part of the regulator. Some well known books that use the Diophantine equation are those by Kučra [34], Åström and Wittenmark [1, 2], Middleton and Goodwin [41] and Chen [15] (this also in the MIMO case). The properties of the exosystem's internal model in the MIMO case were studied by Francis, Sebakhy and Wonham [26, 28], while the most complete solution to the multivariable regulation problem with multiple internal model and complete eigenvalue assignment is due to Francis $[24,25]$ : this solution has been simply translated into geometric terms in the previously reported algorithm. Of course, eigenvalue assignability with the internal model solves the regulator problem from a strictly mathematical viewpoint, but leaves an important practical problem unsolved: robust stability. A significant recent contribution to the regulator problem with robust stability, stated in the same geometric terms that are used herein, has been made by Cevik and Schumacker [14].

\section{Perfect Tracking with Feedforward, Preview and Preaction}

This section completes the geometric approach to regulation theory by adding the second degree of freedom: feedforward. The purpose is to complete the good asymptotic performance of the feedback loop with a satisfactory transient behavior. In the standard approach this is obtained through a suitable choice of a certain number of zeros of the overall transfer function from reference to output, that are freely assignable without any influence on the closed-loop poles. When the plant has some finite delay or is nonminimum-phase the standard causal approach is no longer satisfactory, and resort to special controllers based on preview of the signals to track, is necessary.

\subsection{Model-Reference SISO Continuous-Time Systems}

Let us consider the regulation scheme shown in Fig. 3,a. Note that there is a substantial difference between the regulator of Fig. 1, that implements only the closed-loop stability and asymptotic tracking features, and that of Fig. 3,a, that must also give a good response to the 
input $r$, or, possibly, reproduce it with negligible error: the new regulator has two inputs with independent sets of zeros. To preserve the internal model, the regulator is realized as shown

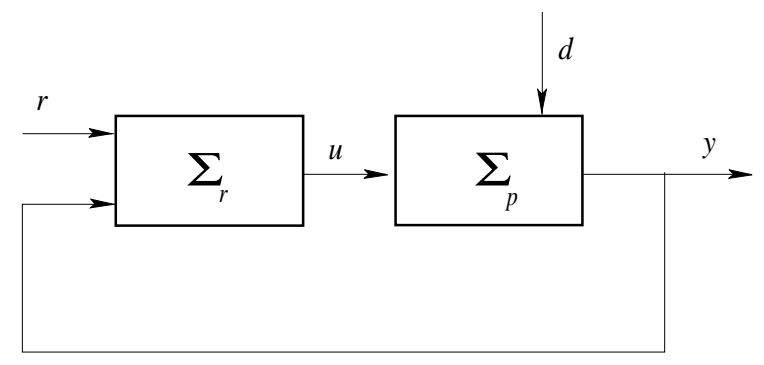

a)

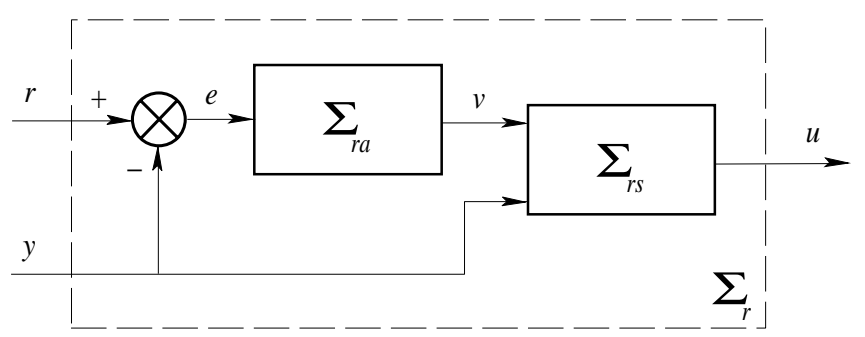

b)

Figure 3: The reference scheme for the two-degrees-of-freedom regulation of continuous-time systems.

in Fig. 3,b, i.e., by interconnection of an asymptotic tracking unit $\Sigma_{r a}$, reproducing only the internal model, and a stabilizing unit $\Sigma_{r s}$, that assign all the closed-loop poles. Let

$$
\frac{P_{r a}(s)}{Q_{r a}(s)}, \frac{P_{r s v}(s)}{Q_{r s}(s)}, \frac{P_{r s y}(s)}{Q_{r s}(s)}, \frac{P_{p}(s)}{Q_{p}(s)}
$$

be the transfer functions of the asymptotic tracking unit, the stabilizing unit with respect to input $v$, the stabilizing unit with respect to input $y$ and the regulated plant respectively. Note that the stabilizing unit has a unique dynamics (hence a unique state-space realization, with two different input distribution matrices).

$Q_{r a}(s)$ depends on the exosystem to track, hence is assumed to be a-priori known, with degree $n_{e}^{\prime}{ }^{11}$ Let $m_{p}$ and $n_{p}$ be the degrees of $P_{p}(s)$ and $Q_{p}(s)$ respectively; the degree of $Q_{r s}(s)$ is $n_{p}$, if the stabilizing unit is realized through a full-order observer, or $n_{p}-1$, if a reduced-order observer is used. Polynomials $Q_{r s}(s)$ and $P_{r s y}(s)$ are determined by assigning the $2 n_{p}$ or $2 n_{p}-1$ poles of the inner loop, while $P_{r a}(s)$ and $P_{r s v}(s)$ are free, with degrees ranging from 0 to $n_{e}^{\prime}$ and from 0 to $n_{p}$ or $n_{p}-1$ respectively. They can be used to implement a model-reference design, where the transfer function $G_{0}(s)=P_{0}(s) / Q_{0}(s)$ from $r$ to $y$ is arbitrarily assigned (with only some generic and easy-to-handle constraints) if the plant is minimum-phase, i.e., with all its zeros stable. This is set in more precise terms in the following Theorem.

Theorem 4.1 (Constraints on the Model-Reference Transfer Function) Let us assume that the plant is minimum-phase. The regulator shown in Fig. 3,b allows reproduction of a modelreference transfer function $P_{0}(s) / Q_{0}(s)$ with $P_{0}(s)$ having degree $m_{0}$ and $Q_{0}(s)$ degree $n_{0}$ under

\footnotetext{
${ }^{11}$ We denote, like in the previous section, by $n_{e}$ the degree of the exosystem and by $n_{e}^{\prime} \leq n_{e}$ that of the internal model, that is less in the case where the plant has some poles equal to those of the exosystem.
} 
the constraints:

- $Q_{\text {ra }}$ is a divisor of $Q_{0}-P_{0}$;

- $m_{0} \geq n_{e}^{\prime}-1$

$-n_{0} \geq m_{0}+n_{p}-m_{p}$.

Proof. Let

$$
H(s):=\frac{P_{r s v}(s) P_{p}(s)}{Q_{c l}(s)} \text { and } H_{1}:=\frac{P_{r a}(s)}{Q_{r a}(s)} H(s)
$$

be the transfer functions of the stabilized plant and the outer loop respectively. Condition (4.2) comes from

$$
\frac{P_{0}(s)}{Q_{0}(s)}=\frac{H_{1}(s)}{1+H_{1}(s)} \text { hence } H_{1}(s)=\frac{P_{0}(s)}{Q_{0}(s)-P_{0}(s)}
$$

By factorization of $P_{0}(s)$ in (4.2) it follows that

$$
Q_{0}(s)=Q_{r a}(s) Q_{0}^{\prime}(s)+P_{0}^{\prime}(s) P_{0}^{\prime \prime}(s)
$$

that can be interpreted as a Diophantine equation with $C(s):=Q_{0}(s), A(s):=Q_{r a}(s)$ and $B(s):=P_{0}^{\prime \prime}(s)$ (given) and $X(s):=Q_{0}^{\prime}(s), Y(s):=P_{0}^{\prime}(s)$ (to be determined). Denote by $l$ the degree of $Q_{0}(s)$ and $l_{1}$ that of $P_{0}^{\prime \prime}(s)$, that is subject to the constraint $l_{1} \leq l-n_{e}^{\prime}$ : it follows that the degree of $Q_{0}^{\prime}(s)$ is $l-n_{e}^{\prime}$ and the degree of $P_{0}^{\prime}(s)$ is $n_{e}^{\prime}-1$. If we assume $Q_{0}^{\prime \prime}(s)$ with minimum degree, i.e., equal to a real constant, (4.3) immediately follows. Relations on the right of (4.5) and (4.6) imply

$$
\frac{P_{r a}(s)}{Q_{r a}(s)}=\frac{P_{0}(s)}{Q_{0}(s)-P_{0}(s)} \frac{Q_{c l}(s)}{P_{r s v}(s) P_{p}(s)}
$$

from which it is easily seen that, if polynomials $P_{r s v}(s)$ and $Q_{c l}(s)$ are assumed to perform the maximum number of pole-zero cancellations, (4.4) holds with the equality sign.

If the plant is nonminumum-phase its unstable zeros cannot be cancelled with corresponding poles in the regulator, so they must be repeated in the model as roots of $P_{0}(s)$, thus seriously conditioning the overall system behavior and making the model reference design questionable. In this case the conditions stated in Theorem 4.1 still hold, provided, of course, that in the member on the right of (4.3) the number of the unstable zeros of the plant is also considered.

It is worth noting that the stabilizing unit $\Sigma_{s}$ may receive more than one feedback signal from the plant to improve robustness of the stabilizing inner loop; in this case the unit uses a reduced-order observer: Theorem 4.1 still holds, since the numbers of the assignable zeros and the assignable poles of $H(s)$ are reduced by the same amount.

\subsection{Preview and Preaction in SISO Discrete-Time Systems}

In the discrete-time case things go significantly better. The reason for this is not inherent in the type of system, but in the mind of control engineers and scientists, who usually accept delays in tracking in the discrete-time case and do not in the continuous-time case. This is a very crucial point because delayed tracking implies preview, hence renouncing causality. Knowing the signal to be tracked a certain time in advance is called preview and acting on the manipulated input of the plant to prepare tracking is called preaction. Preaction time is not necessarily equal to 
preview time: in fact, in some cases tracking with zero error is possible without any preaction. Of course, preaction is not possible without preview and for any input segment to be tracked preaction time must be less than or at most equal to preview time.

It has been shown in the literature that preview and consequent preaction provide substantial benefits in systems with delays (this is obvious) and in nonminimum-phase systems (this may be less apparent). As far as the scenario where preview and preaction give benefits in tracking problems is concerned, it is worth distinguishing the following three cases:

1. infinite preview time and arbitrarily large preaction time (tracking a profile with a machinetool);

2. preview and preaction times variable during operation (tracking a route with an aircraft);

3. preview and preaction times fixed during operation (like in receding-horizon modelpredictive control).

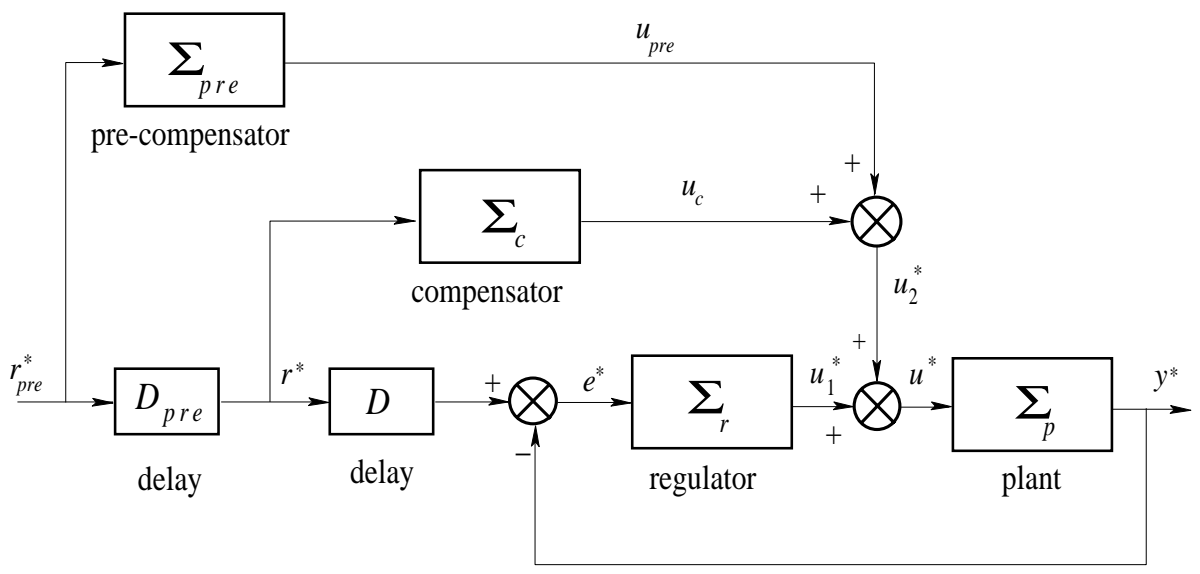

Figure 4: The reference scheme for the two-degrees-of-freedom regulation of discrete-time systems.

In the discrete-time case the objective of control is usually more ambitious: model-reference with $G_{0}(z)=1$, i.e., perfect tracking. The control system in the more general case has the structure shown in Fig. 4: we accept a delay $D$ in tracking, corresponding to the relative degree of the plant, including a possible finite delay, i.e., a multiple pole at $z=0$. Hence, even if the blocks $D_{\text {pre }}$ and $\Sigma_{\text {pre }}$ are absent, there is a preaction through the feedforward compensator $\Sigma_{c}$. This compensator is sufficient to ensure perfect tracking when the plant is minimum-phase. If the plant is nonminimum-phase however a greater preaction time may be required, since in this case the preaction time does not only depend on the plant relative degree, but also on the absolute value of the unstable zeros: in this case a further delay $D_{\text {pre }}$ and a special pre-compensator $\Sigma_{\text {pre }}$ are necessary to obtain almost perfect tracking. Perfect tracking, in the strict mathematical sense, is obtained when delay $D_{\text {pre }}$ is infinity: in practice, almost perfect tracking is achieved if the total preaction time corresponding to $D_{\text {pre }}$ and $D$ is such that the mode corresponding to the unstable zero with absolute value closest to one, considered in the reverse time direction, becomes negligible with respect to its initial value.

The control scheme shown in Fig. 4 implements the receding-horizon control (the third of the previously specified cases). To fit in also the other cases it is necessary to use the more general 


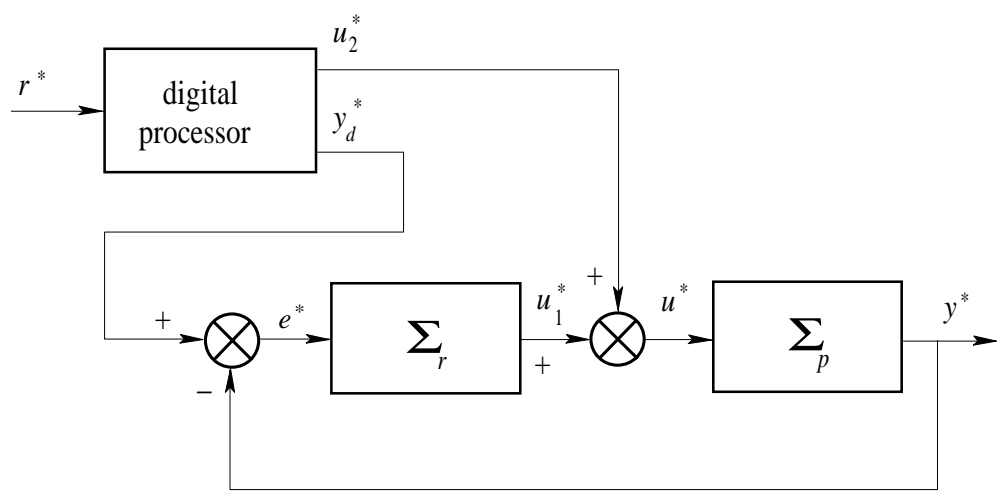

Figure 5: The dual-input controller for discrete-time systems.

implementation shown in Fig. 5, where a digital processor, performing simply the convolution of the previewed part of the reference signal to be tracked with a pre-computed sequence depending on the plant transfer function, directly generates the feedforward signal.

\subsection{Perfect Tracking in SISO Continuous-Time Systems}

We now propose a special control that extends to the continuous-time case all the characterizing features of the dual-input controller shown in Fig. 5. Consider the control system structured as in Fig. 6 consisting of a plant $\Sigma_{p}$, a regulator $\Sigma_{r}$, a compensator $\Sigma_{c}$, a signal generator (or exosystem) $\Sigma_{e}$ and (possibly) a filter $\Sigma_{f}$. The control loop is standard, designed to achieve asymptotic robust tracking of the exosystem signals and disturbance rejection. The supervising unit shown in the figure receives the reference input $r$ and reproduces it, possibly with some delay, by changing the exosystem state at suitable instants of time; it also can change the states of $\Sigma_{r}$ and $\Sigma_{c}$ at any time. The filter $\Sigma_{f}$ is included in the forward path to obtain a reference signal $y_{d}(t)$ smooth enough to be tracked by the plant with piecewise-continuous control input $u(t)$.

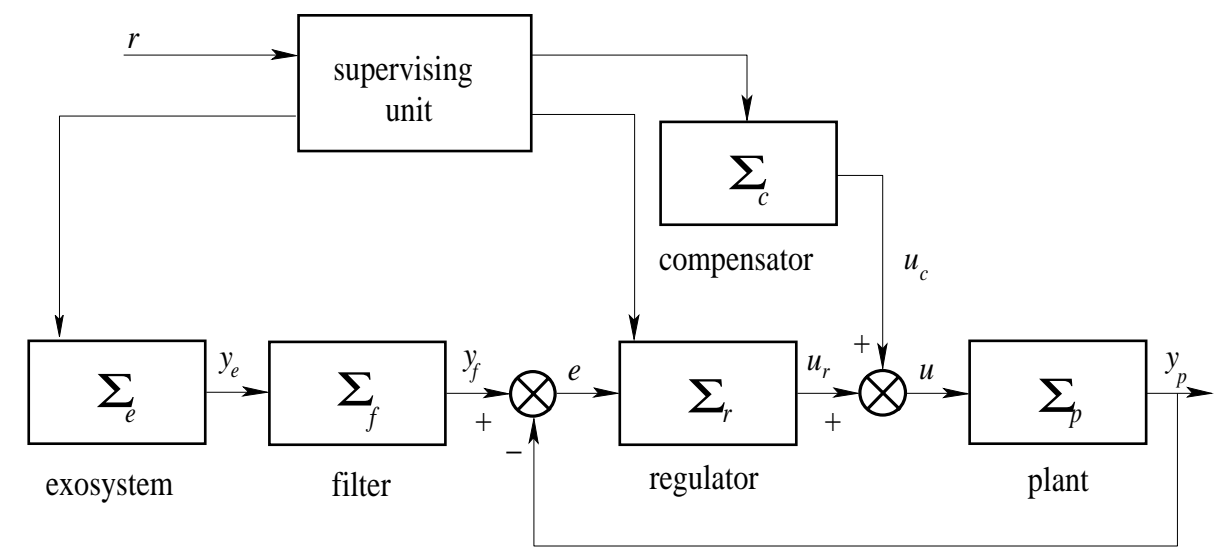

Figure 6: The SISO perfect or almost-perfect tracking implementation.

We assume that the plant, possibly nonminimum-phase, has no zeros on the imaginary axis, and that an internal model of the exosystem is included in the feedforward path of the regulation 
loop. Hence the loop is of the standard type discussed in the previous section. The transfer functions of the regulator and the plant are denoted by

$$
G_{r}(s)=\frac{P_{r}(s)}{Q_{r}(s)}, \quad G_{p}(s)=\frac{P_{p}(s)}{Q_{p}(s)}
$$

The signal generated by $\Sigma_{e}$ is described in terms of its Laplace transform by

$$
Y_{e}(s)=\frac{\alpha}{s^{l}} .
$$

A more realistic signal to be tracked is represented by a time-shifted linear combination of the above signal (see Fig. 7), i.e.,

$$
Y_{e}(s)=\frac{\alpha_{0}}{s^{l}}+\frac{\alpha_{1}}{s^{l}} e^{-t_{1} s}+\ldots+\frac{\alpha_{k}}{s^{l}} e^{-t_{k} s},
$$

where $0<t_{1}<t_{2}<\ldots t_{k}$, for some integer $k$. Due to linearity and time-invariance of our system the solution this case is obtained as a time-shifted linear combination of the solution for (4.10). Therefore, without loss of generality we will assume zero as the unique switching time.

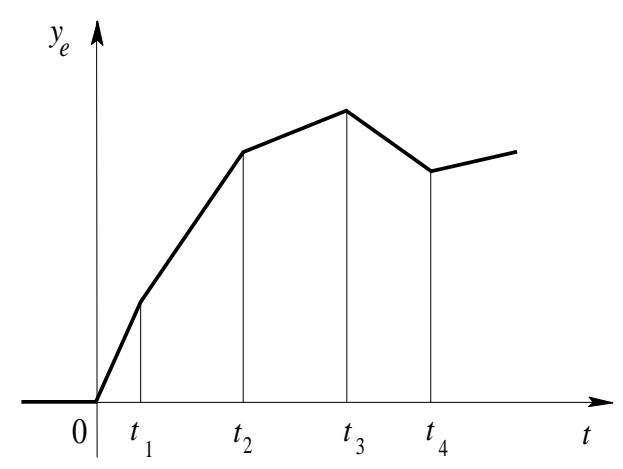

Figure 7: A multiple-switching ramp.

The filter has no zeros and is described by the transfer function

$$
G_{f}(s)=\frac{k_{f}}{Q_{f}(s)}
$$

where $k_{f}$ is a constant and $Q_{f}(s)$, having degree $n_{f}$, is assumed to be strictly stable.

Let $Q_{e}(s):=s^{l}$ : the presence of the internal model implies $Q_{e}(s)=Q_{e}^{\prime}(s) Q_{e}^{\prime \prime}(s)$, with $Q_{e}^{\prime}(s)$ being a divisor of $Q_{r}(s)$ and $Q_{e}^{\prime \prime}(s)$ a divisor of $Q_{p}(s)$. The strictly-proper rational function

$$
U_{r}(s)=\frac{P_{e}(s)}{Q_{e}^{\prime}(s)}
$$

can be considered the free response, due to a nonzero initial condition, of the part of the internal model contained in the regulator.

The signal generated by $\Sigma_{c}$ is described with its Laplace transform

$$
U_{c}(s)=\frac{P_{c}(s)}{Q_{c}(s)}
$$


where $P_{c}(s)$ and $Q_{c}(s)$ will be determined to obtain perfect tracking of the signal $y_{e}(t)$ corresponding to (4.10), under the assumption that filter, regulator and plant are in the zero state at $t=0$. The states of the internal model and compensator can be suddenly changed at any instant of time by the supervising unit. In our framework, referring to Laplace transforms, the action of the supervising unit can be viewed as delivering Dirac impulses to (4.10), (4.13) and (4.14). If the plant is minimum-phase, perfect tracking can easily be performed without any special artifice, as the following theorem states.

Theorem 4.2 (Perfect tracking in the minimum-phase case) Let us assume that $\Sigma_{p}$ is minimum-phase and without any zero on the imaginary axis. The control system of Fig. 6 allows perfect tracking with bounded $u_{c}$ if and only if the following relative degree condition holds:

$$
n-m \leq l+n_{f}-1
$$

Proof. If. It can be easily seen that the tracking error $e$ can be maintained at zero for a suitable value of $n_{f}$ and suitable polynomials $P_{e}(s), P_{c}(s)$ and $Q_{c}(s)$. In fact, from

$$
E(s)=\frac{\alpha}{s^{l}} \frac{k_{f}}{Q_{f}(s)}-\left(\frac{P_{e}(s)}{Q_{e}^{\prime}(s)}+\frac{P_{c}(s)}{Q_{c}(s)}\right) \frac{P_{p}(s)}{Q_{p}(s)}
$$

by assuming $Q_{p}^{\prime}(s):=Q_{p}(s) / Q_{e}^{\prime \prime}(s)$, we obtain

$$
\frac{\alpha k_{f} Q_{p}^{\prime}(s)}{Q_{e}^{\prime}(s) Q_{f}(s) P_{p}(s)}=\frac{P_{e}(s)}{Q_{e}^{\prime}(s)}+\frac{P_{c}(s)}{Q_{c}(s)}
$$

This equality holds if the member on the left is strictly proper, i.e., if (4.15) holds.

Only if. Suppose the control system of Fig. 6 allows perfect tracking, i.e., $y_{f}(t)=y_{p}(t) t \in \mathbf{R}$. But, $y_{f}$ is continuously differentiable at $t=0$ to the order $l+n_{f}-2$. Since the input to the plant is piecewise continuous by assumption, $y_{f}(t)$ is differentiable up to the order of $n-m-1$. Therefore, clearly $l+n_{f}-2 \geq n-m-1$, from which (4.15) follows.

The proposed solution is not feasible in practice if the plant is nonminimum-phase since the compensator, having the zeros of the plant as poles, is unstable, hence injects unbounded functions at the control input $u$. On the other hand, we have a remedy for this: preaction. Let us factorize the numerator of $G_{p}(s)$ as $P_{p}(s)=P_{p}^{-}(s) P_{p}^{+}(s)$, with $P_{p}^{-}(s)$ and $P_{p}^{+}(s)$ having all roots with strictly negative and strictly positive real parts respectively and decompose the last term on the right of (4.17) as

$$
\frac{P_{c}(s)}{Q_{c}(s)}=\frac{P_{c s}(s)}{P_{f}(s) P_{p}^{-}(s)}+\frac{P_{c u}(s)}{P_{p}^{+}(s)}
$$

thus introducing two kinds of open-loop compensators, which are categorized as the postaction (meaning after switching) and preaction (meaning before switching or anticipatory) compensator. We will allow the preaction to start at a suitable instant of time $t_{\text {pre }}<0$. While the postaction compensator is a linear autonomous system whose state can be suitably changed by the supervising unit, the preaction compensator can be realized as a autonomous unstable linear system with a suitable final state and a "small" in norm initial state: its practical implementation is based on recording the sampled backward solution of the state equation and forcing the 
compensator to follow this solution in the forward mode by correcting its state at the sampling instants, considered in the reverse order with respect to the backward solution. In Fig. 8 a typical output signal of the compensator is shown, referring to a case where both preaction and postaction are needed.

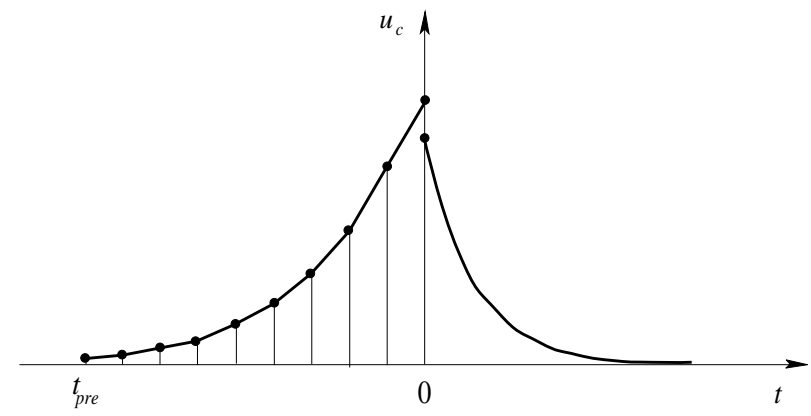

Figure 8: A typical output signal of the compensator, with preaction and postaction.

With the above system description and assumptions we state the main result on SISO almost perfect tracking with preaction in the following terms.

Theorem 4.3 (Almost Perfect Tracking with Preaction) Let us assume that $\Sigma_{p}$ is nonminimum-phase, but with no zeros on the imaginary axis. Denote by $t_{\text {pre }}<0$ the time at which the control action starts, i.e., the smallest $t$ such that $u(t) \neq 0$, and assume that the plant state is zero at $t=t_{\text {pre. }}$. It is possible to realize a control system as shown in Fig. 6 with bounded $u_{c}$ such that

$$
\begin{aligned}
& |e(t)| \rightarrow 0 \text { as } t_{\text {pre }} \rightarrow-\infty \text { uniformly in } \mathbf{R} ; \\
& \int_{-\infty}^{+\infty}|e(t)|^{2} d t \rightarrow 0 \quad \text { as } t_{\text {pre }} \rightarrow-\infty
\end{aligned}
$$

if and only if the relative degree condition (4.15) holds.

Note that (4.19) and (4.20) imply perfect tracking for an infinite preaction time. The main result on almost perfect tracking with preaction is stated as follows.

To prove Theorem 4.3 we need the following lemma.

Lemma 4.1 (Time Axis Inversion) Let us consider a continuous-time causal SISO system $\Sigma$ with transfer function $P(s) / Q(s)$ and denote by $P_{0}(s) / Q(s)$ the Laplace transform of a generic free response of $\Sigma$. Let $u_{r}(t), t \in[-\infty, 0]$, be an input function that, starting from the zero state at $t=-\infty$, takes $\Sigma$ at $t=0$ to the state corresponding to the given free response, and $y_{r}(t)$, $t \in[-\infty, 0]$, the corresponding output function; denote by $U_{r}(s)$ and $Y_{r}(s)$ their reverse-time Laplace transforms, i.e., the Laplace transforms of the functions $u_{r}(-\tau), y_{r}(-\tau), \tau \in[0, \infty]$, defined by

$$
U_{r}(s)=\int_{0}^{\infty} u_{r}(-\tau) e^{-s \tau} d \tau, \quad Y_{r}(s)=\int_{0}^{\infty} y_{r}(-\tau) e^{-s \tau} d \tau
$$

The following relation holds:

$$
Y_{r}(s)=\frac{P(-s)}{Q(-s)} U_{r}(s)-\frac{P_{0}(-s)}{Q(-s)}
$$


with

$$
\frac{P_{0}(-s)}{Q(-s)}:=\left.\frac{P_{0}(s)}{Q(s)}\right|_{s \leftarrow(-s)}, \quad \frac{P(-s)}{Q(-s)}:=\left.\frac{P(s)}{Q(s)}\right|_{s \leftarrow(-s)} .
$$

Proof. Let $(A, b, c, d)$ be a state-space representation of $P(s) / Q(s)$ and $x(0)=x_{0}$ the initial state corresponding to $P_{0}(s) / Q(s)$. It is well known that

$$
\begin{aligned}
\frac{P(s)}{Q(s)} & =c(s I-A)^{-1} b+d, \\
\frac{P_{0}(s)}{Q(s)} & =c(s I-A)^{-1} x_{0},
\end{aligned}
$$

with $(s I-A)^{-1}=\operatorname{adj}(s I-A) / \operatorname{det}(s I-A)$. Functions $u_{r}(\cdot)$ and $y_{r}(\cdot)$ satisfy the state space equations

$$
\begin{aligned}
-\dot{x}_{r}(\tau) & =A x_{r}(\tau)+b u_{r}(\tau), \quad x_{r}(0)=x_{0} \\
y_{r}(\tau) & =c x_{r}(\tau)+d u_{r}(\tau)
\end{aligned}
$$

The corresponding state function is $x_{r}(t), t \in[-\infty, 0]$ (or $x_{r}(-\tau), \tau \in[0, \infty]$ ), whose reverse-time Laplace transform, similar to (4.21), is

$$
X_{r}(s)=\int_{0}^{\infty} x_{r}(-\tau) e^{-s \tau} d \tau
$$

The Laplace transform of (4.26), (4.27) is

$$
\begin{aligned}
-s X_{r}(s)+x_{0} & =A X_{r}(s)+b U_{r}(s) \\
Y_{r}(s) & =c X_{r}(s)+d U_{r}(s)
\end{aligned}
$$

and provides

$$
Y_{r}(s)=\left(c(-s I-A)^{-1} b+d\right) U_{r}(s)-c(-s I-A)^{-1} x_{0}
$$

from which (4.22), (4.23) are derived by comparison with (4.24), (4.25).

Proof of Theorem 4.3. If. Since the control action must be bounded, the postaction signal whose Laplace transform is the last term on the right of (4.18) cannot be applied. We shall show, however, that the same effect can be obtained with a suitable preaction signal. In plain terms, this is proved as follows: since this signal is a linear combination of modes corresponding to zeros of the plant, an initial state of the plant exists that nulls its effect on the output; hence, the opposite of this initial state has the same effect as the signal. It follows that perfect tracking is still obtainable if we are able to reach this initial state with a bounded preaction. Let us consider the equality

$$
\frac{P_{p}(s)}{Q_{p}(s)} \frac{P_{c u}(s)}{P_{p}^{+}(s)}=\frac{P_{p}^{-}(s) P_{c u}(s)}{Q_{p}(s)}
$$

where the term on the right is the equivalent initial state and denote by $u_{c, p r e}(t), t \in[-\infty, 0]$, the preaction signal, that can be derived by using Lemma 4.1. Let $U_{c, p r e}(s)$ be the Laplace transform of the reverse-time function $u_{c, p r e}(-\tau), \tau \in[0, \infty]$. From (4.22) that in this case becomes

$$
Y_{r}(s)=\frac{P_{p}(-s)}{Q_{p}(-s)} U_{c, p r e}(s)-\frac{P_{p}^{-}(-s) P_{c u}(-s)}{Q_{p}(-s)}
$$


we obtain that the preaction signal whose reverse-time Laplace transform is

$$
U_{c, p r e}(s)=\frac{P_{c u}(-s)}{P_{p}^{+}(-s)}
$$

takes the plant to the desired state while maintaining the output identically zero. Thus error can be nulled if $t_{\text {pre }} \rightarrow-\infty$. The convergence properties (4.19) and (4.20) will be proved for the MIMO case in Theorem 4.5 of the next subsection.

Only if. This part of the proof is the same as for Theorem 4.2.

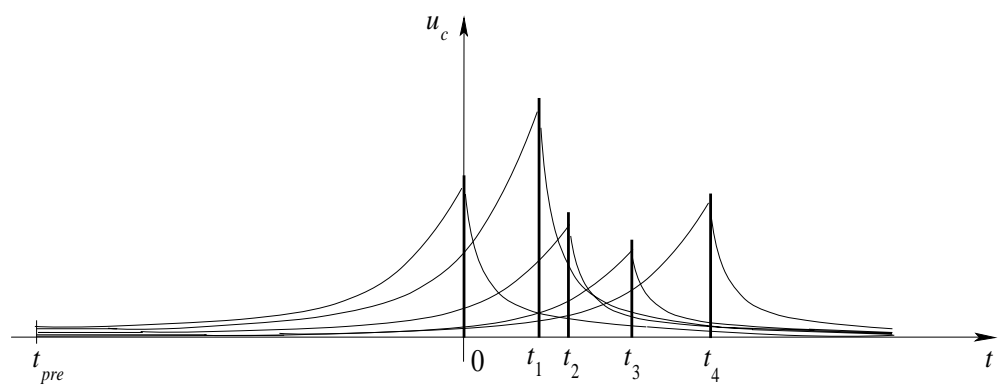

Figure 9: A typical correction signal in the case of infinite preview.
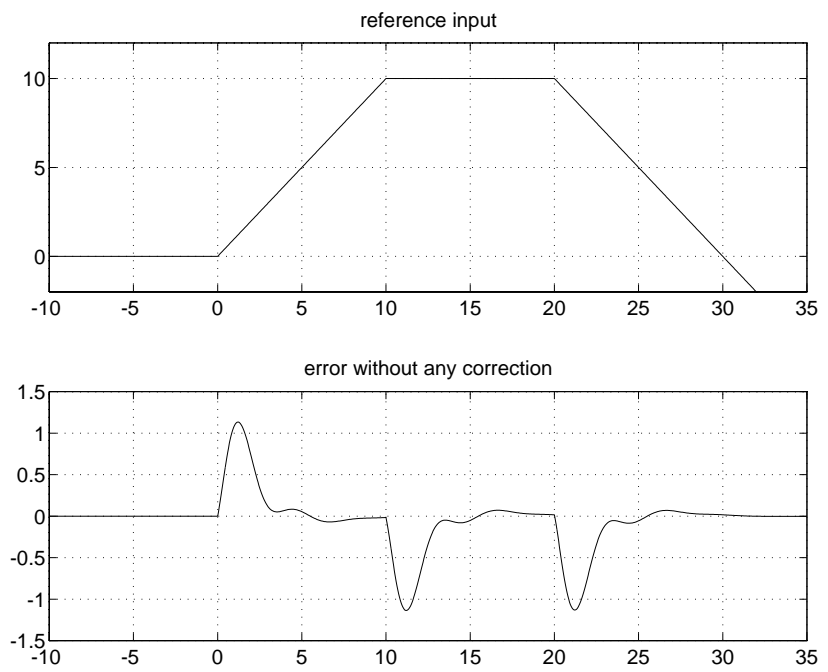

Figure 10: The behavior of the control loop without any correction.

\section{Remarks:}

1. A delay $e^{-t_{0} s}$ in the plant can be easily handled by the supervising unit by advancing all the correcting signal to the regulator and compensator with respect to the reference.

2. Preaction time is not necessarily equal to preview time. For instance, consider a machinetool that must reproduce a profile completely known in advance: the preview horizon is 

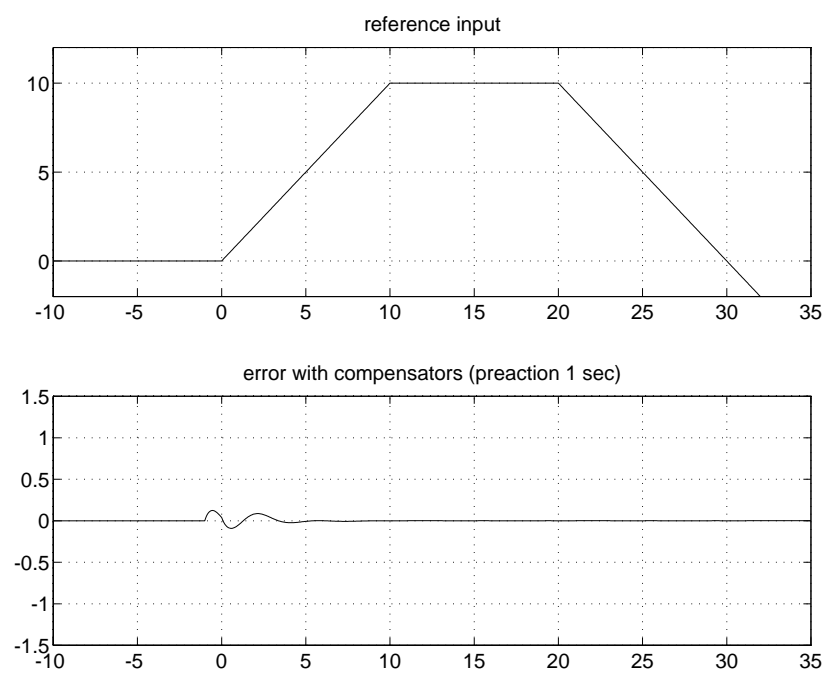

Figure 11: The behavior of the control system with $t_{\text {pre }}=-1$.

infinity, since we can impose any movement after the profile has been followed without error (for instance, a permanent stop condition), while preaction time has to be chosen to neutralize the effects of the unstable zeros of the plant, and thus is related to the maximum time constant corresponding to their real parts. In this case if error is zero at the first exosystem switching, it remains zero during all tracking since in preaction all future switchings have been accounted for. This is clarified by Fig. 9, where the correcting pulses of both preaction and postaction for an infinite preview horizon is shown: since the preaction time increases, perfect tracking is swiftly achieved, after a unique small initial transient.

\subsection{A Numerical Example}

The above concepts will be illustrated by a simple numerical example. Let

$$
G_{p}(s)=\frac{-3(s-2)(s+4)}{(s+1)(s+2)(s+8)}
$$

be the transfer function of the plant, and

$$
G_{r}(s)=\frac{(s+.5)^{2}+.3^{2}}{s^{2}}
$$

that of a standard regulator with a double pole at the origin, required to track a ramp with asymptotic zero error. In this case according to the relative degree condition a filter is not required to follow multiple-switching ramps of the types shown in Fig. 7.

Fig. 10 shows the reference input and the error of the standard control loop. Fig. 11 shows the error, the regulator output and the compensator output corresponding to the overall implementation scheme represented in Fig. 6 with $t_{p r e}=-1$ sec. The supervising unit in this 

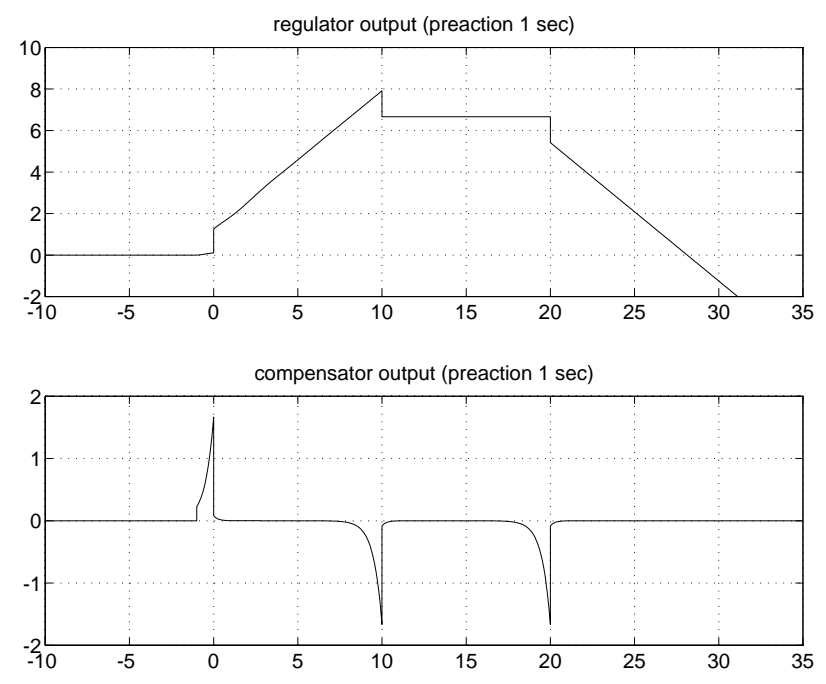

Figure 12: The outputs of the regulator and compensator with $t_{\text {pre }}=-1$.

case drives the unstable zero compensator state along the unstable trajectory starting a $t_{\text {pre }}$ (by imposing backward-computed samples, as shown in Fig. 8) and changes the internal model and stable zero compensator states at the switching times. Note that the error transient is significantly reduced and is present only at the first switching, since an infinite preview horizon has been assumed; if the preview horizon was equal to the preaction time (i.e., switching times were known only one second in advance), the same error transient would be present at every switching. Fig. 12 shows the regulator and compensator output in the same case.

The preaction time $t_{\text {pre }}=-5 \mathrm{sec}$ reduces the error to practically zero (its maximum absolute value during transient after $t_{\text {pre }}$ is about $10^{-4}$ ) and maintains it at zero at every switching time.

\subsection{Perfect Tracking in MIMO Continuous-Time Systems}

The control system layout of Fig. 6 is repeated in Fig. 13 for the MIMO case: it includes a standard multivariable feedback loop consisting of a plant $\Sigma_{p}$ and a regulator $\Sigma_{r}$, designed to react to and asymptotically reject inaccessible and unpredictable disturbances possibly applied to the plant and not shown in the figure. The scheme also includes a supervising unit whose aim is to realize, if possible, tracking with zero error of vector reference input $r(t)=\left(r_{1}(t), \ldots, r_{q}(t)\right)$ by suitably killing tracking error transients in the feedback loop.

To this end the supervising unit: (i) generates an approximate replica of the reference input by changing, at discrete instants of time, the leading states of the exosystems $\Sigma_{e, i}$, each followed by a filter $\Sigma_{f, i}(i=1, \ldots, q)$, whose purpose is to apply sufficiently smooth signals to the regulation loop; (ii) reads and changes the regulator state when necessary; (iii) changes the state of a feedforward compensator $\Sigma_{c}$ when necessary, thus generating correcting signals that are injected on the manipulable input of the plant.

In practice the regulator, exosystems, filters, feedforward compensator and supervising unit can be realized as a single special-purpose digital computer. However, for a neater presentation 


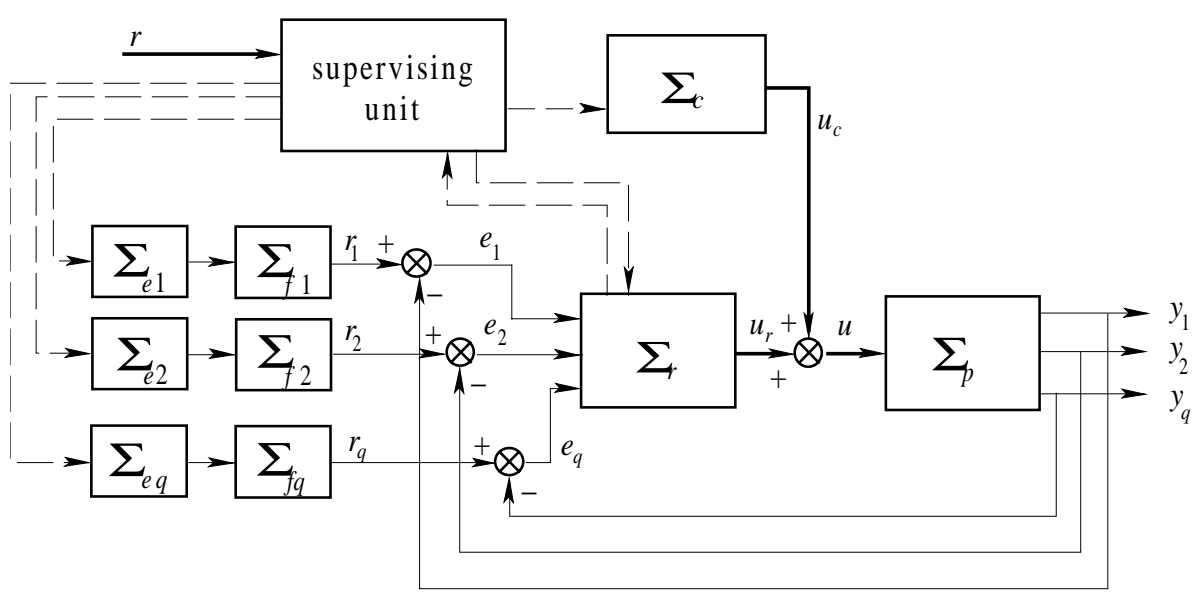

Figure 13: The MIMO perfect or almost-perfect tracking implementation.

of the results, in this paper continuous-time models will be adopted.

The plant $\Sigma_{p}$ is modelled by the triple $\left(A_{p}, B_{p}, C_{p}\right)$ with state dimension $n_{p}$, and is assumed to be functionally controllable, i.e., according to Property 2.9, satisfying

$$
\mathcal{S}^{*}+\mathcal{C}=\mathcal{X}_{p}
$$

with $\mathcal{S}^{*}:=\min \mathcal{S}\left(A_{p}, B_{p}, C_{p}\right), \mathcal{C}:=\operatorname{ker} C_{p}$, and $\mathcal{X}_{p}:=\mathbf{R}^{n_{p}}$.

If (4.37) holds, by using Algorithm 2.10 it is possible to define a relative degree $\rho_{i}$, $(i=1, \ldots, q)$, referred to the outputs of the plant.

We assume that the generic $i$-th exosystem, of order $n_{e, i}$, is represented by the observable pair $\left(C_{e, i}, A_{e, i}\right)(i=1, \ldots, q)$, with

$$
A_{e, i}=\left[\begin{array}{cccc}
0 & 1 & \ldots & 0 \\
0 & 0 & \ldots & 0 \\
\vdots & \vdots & \ddots & \vdots \\
-\eta_{i, 0} & -\eta_{i, 1} & \ldots & -\eta_{i, n_{e, i}-1}
\end{array}\right], \quad C_{e, i}=\left[\begin{array}{llll}
1 & 0 & \ldots & 0
\end{array}\right]
$$

The filters are represented by strictly stable triples $\left(A_{f, i}, B_{f, i}, C_{f, i}\right)$, of orders $n_{f, i}$ $(i=1, \ldots, q)$. Like the exosystems, the filters are given in the observer canonical form, i.e.,

$$
\begin{aligned}
A_{f, i} & =\left[\begin{array}{cccc}
0 & 1 & \ldots & 0 \\
0 & 0 & \ldots & 0 \\
\vdots & \vdots & \ddots & \vdots \\
-\varphi_{i, 0} & -\varphi_{i, 1} & \ldots & -\varphi_{i, n_{f, i}-1}
\end{array}\right], B_{f, i}=\left[\begin{array}{c}
0 \\
0 \\
\vdots \\
\varphi_{i, 0}
\end{array}\right], \\
C_{f, i} & =\left[\begin{array}{cccc}
1 & 0 & \ldots & 0
\end{array}\right]
\end{aligned}
$$

that guarantees absence of zeros, hence relative degree $n_{f, i}$.

It may happen that some filters are not necessary in the overall system represented in Fig. 13. To include also this case we represent filters with quadruples $\left(A_{f, i}, B_{f, i}, C_{f, i}, D_{f, i}\right)$ with the agreement that $D_{f, i}=1$ if the other matrices are empty and $D_{f, i}=0$ otherwise. The overall filtering system can be represented by the quadruple $\left(A_{f}, B_{f}, C_{f}, D_{f}\right)$, where matrices $A_{f}$ and $D_{f}$ are block-diagonal built with those of the single filters, while $B_{f}$ and $C_{f}$ are built in the same 
way, but completed with zero columns or rows to save dimensional congruence when dynamics is absent.

For the multivariable loop we use the standard state-space representation whose equations are of the type (3.15), (3.17), that model the plant, filters and exosystem as a unique triple $(A, B, E)$

It is possible to include filters in matrix $A$ of (3.15) by redefining the submatrices (3.16) as

$$
\begin{aligned}
x_{1} & :=\left[\begin{array}{l}
x_{p} \\
x_{f}
\end{array}\right], \quad A_{1}:=\left[\begin{array}{cc}
A_{p} & O \\
O & A_{f}
\end{array}\right], \quad A_{3}:=\left[\begin{array}{c}
O \\
B_{f} C_{e}
\end{array}\right], \quad B_{1}:=\left[\begin{array}{c}
B_{p} \\
O
\end{array}\right] \\
x_{2} & :=x_{e}, \quad E_{1}:=\left[\begin{array}{ll}
-C_{p} & C_{f}
\end{array}\right], \quad E_{2}:=D_{f} C_{e}
\end{aligned}
$$

so that $x_{1} \in \mathbf{R}^{n_{1}}$, with $n_{1}=n_{p}+n_{f}$, now denotes the state of the system "plant plus filters". Note that $\left(A_{1}, B_{1}\right)$ is still stabilizable, $(A, E)$ detectable, and (3.36), (3.37) still hold. Furthermore, the regulator designed for the plant in the absence of filters still satisfies both the asymptotic regulation condition and the loop stability condition, since the modes of the filters tend to zero as time approaches infinity, like those of the control loop.

The feedforward compensator is represented by the observable pair $\left(C_{c}, A_{c}\right)$, of order $n_{c}$. We state the following theorem, that extends Theorem 4.2 to the MIMO case.

Theorem 4.4 (Perfect tracking in the minimum-phase case) Assume that (i) $\Sigma_{p}$ is functionally controllable, (ii) $\Sigma_{p}$ is minimum-phase (i.e., $\left.\mathcal{Z}\left(A_{p}, B_{p}, C_{p}\right) \subseteq \mathbf{C}_{-}\right)$. The control system of Fig. 13 allows perfect tracking with bounded $u_{c}$ if and only if the following relative degree condition holds:

$$
n_{f, i} \geq \rho_{i}-n_{e, i}+1 \quad(i=1, \ldots, q)
$$

Proof. If. Let $x_{e, i}(0+)=\left(0,0, \ldots, \alpha_{i}\right)^{T}(i=1, \ldots, q)$ be the column vectors of the initial states of the exosystems and denote with $x_{2}(0+)$ the overall exosystem initial state, a column vector having these vectors as elements. Consider the basis matrix (3.25) for $\hat{\mathcal{W}}$ referring to the regulator problem with matrices defined by (4.40), (4.41), i.e., with filters modelled as an uncontrollable part of the plant. The overall system initial state corresponding to tracking without transients is computed as

$$
\left[\begin{array}{c}
x_{1}(0+) \\
x_{2}(0+) \\
z(0+)
\end{array}\right]=\left[\begin{array}{c}
X_{1} \\
I_{n_{2}} \\
Z
\end{array}\right] x_{2}(0+) .
$$

Since the relative degree condition (4.42) is satisfied, perfect tracking by means of a suitable control function is possible. This implies that state $x_{1}(0+)$ belongs to an $\left(A_{1}, \mathrm{im} B_{1}\right)$-controlled invariant contained in $\operatorname{ker} E_{1}$, hence to $\mathcal{V}_{1}^{*}=\max \mathcal{V}\left(A_{1}, \operatorname{im} B_{1}, \operatorname{ker} E_{1}\right)$, since only if this is so does a corrective control function $u_{c}(t)$ exist that, starting from $-x_{1}(0+)$, maintains the plant state trajectory on $\mathcal{V}_{1}^{*}$, hence with no influence on the error variable. By superposition, this control function keeps the error variable at zero if applied with our actual initial state $\left(0, x_{2}(0+), z(0+)\right)^{T}$. It is easily shown that $u_{c}(t)$ can be generated by the compensator: let us denote by $n_{c}$ the dimension of $\mathcal{V}_{1}^{*}$ and by $T_{1}$ a $n_{1} \times n_{c}$ basis matrix of $\mathcal{V}_{1}^{*}$. Let $F_{1}$ be a matrix such that $\left(A_{1}+B_{1} F_{1}\right) \mathcal{V}_{1}^{*} \subseteq \mathcal{V}_{1}^{*}$, define $T=\left[T_{1} T_{2}\right]$ with $T_{2}$ such that $T$ is nonsingular and assume as $A_{c}$ the first $n_{c} \times n_{c}$ submatrix of $T^{-1}\left(A_{1}+B_{1} F_{1}\right) T$ and as $C_{c}$ the $p \times n_{c}$ matrix $F_{1} T_{1}$. The initial state $x_{c}(0+)$ of the compensator is then given by the first $n_{c}$ components of $-T^{-1} x_{1}(0+)$. The internal unassignable eigenvalues of $\mathcal{V}_{1}^{*}$ are the union of the elements of $\mathcal{Z}\left(A_{p}, B_{p}, C_{p}\right)$ (the invariant zeros of the plant) 
and of the eigenvalues of the filters, that appear as invariant zeros of $\left(A_{1}, B_{1}, E_{1}\right)$ since filters are not controllable. Hence the corresponding eigenstructures are repeated in $A_{c}$.

Only if. We suppose that the supervising unit reproduces the reference signal by changing the first state variable of the integrator chain present in every exosystem at discrete instants of time, so that the output of the $i$-th exosystem has the $\left(n_{e, i}-1\right)$-th time derivative piecewise continuous, while the $i$-th output can reproduce any function with $\rho_{i}$-th derivative piecewise continuous, so that a filter satisfying (4.42) is necessary to restrict the class of reference functions.

Remark: The role of the compensator is to drive the state of the plant and filters along trajectories on the $\left(A_{1}+B_{1} F_{1}\right)$-invariant $\mathcal{V}_{1}^{*}$, thus making it possible to reproduce in the controlled plant the effect of state feedback matrix $F_{1}$ for every initial state belonging to $\mathcal{V}_{1}^{*}$. Owing to Theorem 4.4 it is possible to obtain an asymptotically stable compensator if and only if the plant is minimum phase. Hence condition (4.42) is sufficient to ensure zero tracking error of the replicated reference signal with bounded input. Note that $F_{1}$ can and must also stabilize the internal assignable eigenvalues of $\mathcal{V}_{1}^{*}$ if the controlled plant is not invertible.

Theorem 4.5 (Almost Perfect Tracking with Preaction) Assume that (i) $\Sigma_{p}$ is functionally controllable, (ii) $\mathcal{Z}\left(A_{p}, B_{p}, C_{p}\right) \cap \mathbf{C}_{0}=\emptyset$. Denote by $t_{\text {pre }}<0$ the time at which the control action starts, i.e., the smallest $t$ such that $u(t) \neq 0$, and assume that the plant state is zero at $t=t_{\text {pre }}$. It is possible to realize a control system as shown in Fig. 13 with bounded $u_{c}$ such that

$$
\begin{aligned}
& \|e(t)\| \rightarrow 0 \text { as } t_{\text {pre }} \rightarrow-\infty \text { uniformly in } \mathbf{R} \\
& \int_{-\infty}^{+\infty}\|e(t)\|^{2} d t \rightarrow 0 \text { as } t_{\text {pre }} \rightarrow-\infty
\end{aligned}
$$

if and only if the relative degree condition (4.42) holds.

Proof. If. Since $\mathcal{Z}\left(A_{p}, B_{p}, C_{p}\right)$ has no purely imaginary elements, of $A_{c}$ can be partitioned into a strictly stable set and a strictly unstable set. A similarity transformation $T_{c}$ exists such that

$$
T_{c}^{-1} A_{c} T_{c}=\left[\begin{array}{cc}
A_{c, s} & O \\
O & A_{c, u}
\end{array}\right]
$$

where $A_{c, s}$ has the strictly stable eigenvalues and $A_{c, u}$ those strictly unstable. Without loss of generality, we can assume that transformation $T_{c}$ is included in $T_{1}$, so that $A_{c}$ has the structure shown on the right of (4.46) and the initial state $x_{c}(0+)$ of the compensator is accordingly partitioned as $x_{c, s}(0+), x_{c, u}(0+)$. Let

$$
x_{1, s}(0+):=T_{1}\left[\begin{array}{c}
x_{c, s}(0+) \\
0
\end{array}\right], \quad x_{1, u}(0+):=T_{1}\left[\begin{array}{c}
0 \\
x_{c, u}(0+)
\end{array}\right]
$$

as far as $x_{c, s}(0+)$ is concerned, we proceed as before, generating a control action $u_{2}(t)$ corresponding to an output-invisible state trajectory $x_{1}(t)$ of the plant and filters starting from $-x_{1, s}(0+)$ and tending to zero as time approaches infinity, while for $x_{c, u}(0+)$ we integrate the compensator equation backward in time, i.e., with respect to the time variable $\tau:=-t$, thus generating again a stable trajectory $x_{1}(\tau)$ and a stable control function $u_{2}(\tau)$, tending to zero as $\tau$ approaches infinity. Since the reverse-time trajectory $x_{1}(t)=x_{1}(-\tau), t \in[-\infty, 0]$ is admissible and externally stable because of the strict stability of the control loop, by applying the control 
function $u_{2}(t)=u_{2}(-\tau), t \in[-\infty, 0]$ we obtain a zero-output trajectory that reaches $x_{1, u}(0+)$ at $t=0$.

Let us denote by $t_{\text {pre }}<0$ the time at which the control action starts (i.e., the smallest $t$ such that $u(t) \neq 0)$, and assume that the overall system state is zero at $t=t_{\text {pre }}$. Tracking error would be zero if the state of the plant (including filters) at $t=t_{\text {pre }}$ were

$$
x_{1}\left(t_{\text {pre }}\right)=T_{1} e^{A_{c, u} t_{\text {pre }}}\left[\begin{array}{c}
0 \\
x_{c, u}(0+)
\end{array}\right]
$$

but, since the control action starts at $t_{\text {pre }}$, it is zero. The error transient can be computed by considering the response to $-x_{1}\left(t_{\text {pre }}\right)$ of the extended plant (that, in this case, consists of plant, filters and regulator) with the regulator state $z\left(t_{\text {pre }}\right)$ at zero. Let us denote with $\alpha=\left(\alpha_{1}, \ldots, \alpha_{q}\right)$ the vector of the exosystems initial states and let $\sigma_{1}(>0)$ be the minimal real part of the eigenvalues of $A_{c, u}$; since $x_{c, u}(0+)$ is a linear function of $\alpha$, a constant $\delta_{1}>0$ exists such that

$$
\left\|x_{1}\left(t_{\text {pre }}\right)\right\| \leq \delta_{1} e^{\sigma_{1} t_{\text {pre }}}\|\alpha\|
$$

Let

$$
A_{e p}:=\left[\begin{array}{cc}
A_{1}+B_{1} K E_{1} & B_{1} L \\
M E_{1} & N
\end{array}\right], \quad E_{e p}:=\left[\begin{array}{ll}
E_{1} & O
\end{array}\right]
$$

be the matrices of the extended plant. The error is given by

$$
e\left(t-t_{\text {pre }}\right)=E_{\text {ep }} e^{A_{e p}\left(t-t_{\text {pre }}\right)}\left[\begin{array}{c}
-x_{1}\left(t_{\text {pre }}\right) \\
0
\end{array}\right], \quad t \geq t_{\text {pre }}
$$

Let us denote by $\sigma_{2}(<0)$ the maximal real part of the eigenvalues of $A_{e p}$ : a constant $\delta_{2}>0$ exists such that

$$
\begin{aligned}
\|e(t)\| & \leq \delta_{2} e^{\sigma_{2}\left(t-t_{\text {pre }}\right)}\left\|x_{1}\left(t_{\text {pre }}\right)\right\| \leq \delta_{1} \delta_{2} e^{\sigma_{2}\left(t-t_{\text {pre }}\right)} e^{\sigma_{1} t_{\text {pre }}}\|\alpha\| \\
& \leq \delta_{1} \delta_{2} e^{\sigma_{1} t_{\text {pre }}}\|\alpha\|
\end{aligned}
$$

Furthermore,

$$
\begin{aligned}
\int_{-\infty}^{\infty}\|e(t)\|^{2} d t=\int_{t_{\text {pre }}}^{\infty}\|e(t)\|^{2} d t & \leq \int_{t_{\text {pre }}}^{\infty} \delta_{1}^{2} \delta_{2}^{2} e^{2 \sigma_{1}\left(t-t_{\text {pre }}\right)} e^{2 \sigma_{1} t_{\text {pre }}}\|\alpha\| d t \\
& =\frac{\delta_{1}^{2} \delta_{2}^{2}}{-2 \sigma_{2}} e^{2 \sigma_{1} t_{\text {pre }}}\|\alpha\|
\end{aligned}
$$

The statement directly follows from (4.52) and (4.53).

Only if. This part of the proof is as for Theorem 4.4.

Corollary 4.1 Let $\alpha=\left(\alpha_{1}, \ldots, \alpha_{q}\right)$ be the vector of the exosystems initial states. Then, given any number $\epsilon>0$, we have the following:

$$
\begin{aligned}
& t_{\text {pre }} \leq \frac{1}{\sigma_{1}} \ln \frac{\epsilon}{\delta_{1} \delta_{2}\|\alpha\|} \quad \text { implies } \quad\|e(t)\| \leq \epsilon, \quad t \in \mathbf{R} \\
& t_{\text {pre }} \leq \frac{1}{2 \sigma_{1}} \ln \frac{-2 \epsilon \sigma_{2}}{\delta_{1}^{2} \delta_{2}^{2}\|\alpha\|} \quad \text { implies } \quad \int_{-\infty}^{+\infty}\|e(t)\|^{2} d t \leq \epsilon
\end{aligned}
$$

where the quantities $t_{\text {pre }}, \sigma_{1}, \sigma_{2}, \delta_{1}, \delta_{2}$ and $e(t)$ are defined as in the proof of Theorem 4.5. 


\section{Remarks:}

1. The proofs of Theorems 4.4 and 4.5 suggest a working algorithm for the supervising unit to deal with exosystems state switching at $t=0$. Since the overall system is linear and time-invariant, multiple switchings at different instants of time are easily handled by superposition and time-shifting. Note that the regulator state corresponding to tracking without error must be superimposed on the current regulator state, that may be affected by disturbances to be neutralized; hence at every switching the supervising unit must read the current regulator state and apply to it the increment computed with the above algorithm. The same applies to the compensator, whose state, subject to multiple transients, is incremented at every switching by the amount derived by means of the algorithm.

2. The proposed control scheme realizes perfect or almost-perfect tracking control in multivariable systems by injecting suitable correcting pulses at the control input: of course, perfect tracking implies noninteraction, hence it is shown that noninteraction is a seconddegree-of-freedom property.

Many standard problems of multivariable control theory for which a geometric solution was already given, can be revisited with the new concept of preaction, that allows trading causality for stabilizability. An example is given in the following exercise.

Exercise 4.1 Let us refer to the disturbance localization problem (Section 2.6) and assume that the disturbance $d(\cdot)$ is accessible and completely known a priori. Show that the accessible disturbance localization problem with stability has a solution if and only if $(A, B)$ is stabilizable and (2.43) holds.

Notes and References. The model-reference two-degrees-of-freedom method to deal with tracking problems, both in continuous and in discrete-time cases, is well-known. It is also widely used in adaptive control systems (see for instance Åström and Wittenmark [1, 2] and Mosca [45]). It is also known that in LQ optimal tracking and in model-predictive control, preview gives significant benefits in the nonminimum-phase case. Important results in discrete-time control systems using preaction by special design of the feedforward path of a two-degrees-of-freedom controller were obtained by Jayasuriya and Tomizuka [33] and Gross, Tomizuka and Messner [29]. The use of a two-input controller with a special digital unit to deal with the infinite or time-varying preview case in the discrete-time case has been proposed by Marro and Fantoni [37]. In the continuous-time case basic results on system inversion and control with preaction, based on the backward computation of the unstable zeros dynamics, have been presented by Devasia, Paden and Rossi [22, 23] and Hunt, Meyer and Su [31]. The use of a supervising unit feeding compensators that generate correcting pulses at discrete instants of time was applied by Marro and Piazzi to eliminate regulation transients in the presence of large parameter jumps or structural changes in minimum-phase multivariable controlled plants [39].

\section{References}

[1] Åström, K.J., and B. Wittenmark, Computer-Controlled Systems: Theory and Design, Prentice Hall, Englewood Cliffs, N.J., 1990. 
[2] Åström, K.J., and B. Wittenmark, Adaptive Control (2nd edition, Addison-Wesley, Reading, Massachusetts, 1995.

[3] Basile, G., Laschi, R. and G. Marro, "Invarianza controllata e non interazione nello spazio degli stati," L'Elettrotecnica, vol. LVI, n. 1, 1969.

[4] Basile, G., and G. Marro, "Controlled and conditioned invariant subspaces in linear system theory," J. Optimiz. Th. Applic., vol. 3, no. 5, pp. 305-315, 1969.

[5] Basile, G., and G. Marro, "On the observability of linear time-invariant systems with unknown inputs," J. of Optimiz. Th. Applic., vol. 3, no. 6, pp. 410-415, 1969.

[6] Basile, G., and G. Marro, "L'invarianza rispetto ai disturbi studiata nello spazio degli stati," Rendiconti della LXX Riunione Annuale AEI, paper 1-4-01, Rimini, Italy, 1969.

[7] Basile, G., and G. Marro, "A state space approach to noninteracting controls," Ricerche di Automatica, vol. 1, no. 1, pp. 68-77, 1970.

[8] Basile, G., and G. Marro, "On the perfect output controllability of linear dynamic systems," Ricerche di Automatica, vol. 2, no. 1, pp. 1-10, 1971.

[9] Basile, G., and G. Marro, "Self-bounded controlled invariant subspaces: a straightforward approach to constrained controllability," J. Optimiz. Th. Applic., vol. 38, no. 1, pp. 71-81, 1982.

[10] Basile, G., and G. Marro, "On the robust controlled invariant," Systems $\& 3$ Control Letters, vol. 9, no. 3, pp. 191-195, 1987.

[11] Basile, G. and G. Marro, Controlled and Conditioned Invariants in Linear System Theory, Prentice Hall, New Jersey, 1992.

[12] Basile, G., G. Marro, and A. Piazzi, "A new solution to the disturbance localization problem with stability and its dual," Proceedings of the '84 International AMSE Conference on Modelling and Simulation, vol. 1.2, pp. 19-27, Athens, 1984.

[13] Basile, G., G. Marro, and A. Piazzi, "Stability without eigenspaces in the geometric approach: some new results," Frequency Domain and State Space Methods for Linear Systems, edited by C. A. Byrnes and A. Lindquist, North-Holland (Elsevier), Amsterdam, pp. 441$450,1986$.

[14] Cevik, M.K.K. and J.M. Schumacher, "The regulator problem with robust stability," $A u$ tomatica, vol. 31, pp. 1393-1406, 1995.

[15] Chen, C.T., Linear System Theory and Design, Holt, Rinehart and Winston, New York, 1984.

[16] Davison, E.J., "The robust control of a servomechanism problem for linear time-invariant multivariable systems," IEEE Trans. on Autom. Contr., no. 21, pp. 25-33, 1976. 
[17] Davison, E.J., and I.J. Ferguson, "The design of controllers for the multivariable robust servomechanism problem using parameter optimization methods," IEEE Trans. on Autom. Contr., vol. AC-26, no. 1, pp. 93-110, 1981.

[18] Davison, E.J., and A. Goldemberg, "Robust control of a general servomechanism problem: the servo compensator," Automatica, vol. 11, pp. 461-471, 1975.

[19] Davison, E.J., and B.M. Scherzinger, "Perfect control of the robust servomechanism problem," IEEE Trans. on Autom. Contr., vol. AC-32, no. 8, pp. 689-701, 1987.

[20] Davison, E.J., and S.H. Wang, "Properties and calculation of transmission zeros of linear multivariable systems," Automatica, vol. 10, pp. 643-658, 1974.

[21] Davison, E.J., and S.H. Wang, "Remark on multiple transmission zeros" (correspondence item), Automatica, vol. 10, pp. 643-658, 1974.

[22] Devasia, S. and B. Paden, "Exact output tracking for nonlinear time-varying systems", Proc. IEEE Conf. on Decision and Control, Lake Buena Vista, CA, pp. 2346-2355, 1994.

[23] Devasia, S., B. Paden, and C. Rossi, "Minimal transient regulation of flexible structures." Proceedings of the IECON '94, Bologna, Italy, Sept. 5-9 1994.

[24] Francis, B.A., "The linear multivariable regulator problem," SIAM J. Contr. Optimiz., vol. 15, no. 3, pp. 486-505, 1977.

[25] Francis, B.A., "The multivariable servomechanism problem from the input-output viewpoint," IEEE Trans. Autom. Contr., vol. AC-22, no. 3, pp. 322-328, 1977.

[26] Francis, B., O.A. Sebakhy, and W.M. Wonham, "Synthesis of multivariable regulators: the internal model principle," Applied Math. \& Optimiz., vol. 1, no. 1, pp. 64-86, 1974.

[27] Francis, B.A., and W.M. Wonham, "The role of transmission zeros in linear multivariable regulators," Int. J. Control, vol. 22, no. 5, pp. 657-681, 1975.

[28] Francis, B.A., and W.M. Wonham, "The internal model principle of control theory," Automatica, no. 12, pp. 457-465, 1976.

[29] Gross, E., M. Tomizuka and W. Messner, "Cancellation of discrete time unstable zeros by feedforward control," ASME J. Dynamic Syst., Measurement Contr., vol. 116, no. 1, pp. 33-38, Mar. 1994.

[30] Hamano, F. and G. Marro, "Using preaction to eliminate tracking error in feedback control of multivariable systems," DEIS Report no. GA-3-94, Università di Bologna, Italy, 1994 (also submitted to CDC'96, Osaka, Japan).

[31] Hunt, L.R., G. Meyer and R. Su, "Output tracking for nonlinear systems," Symposium on Implicit and Nonlinear Systems, Arlington, Texas, pp. 314-320, 1992.

[32] Isidori, A., Nonlinear Control Systems (3rd edition), Springer-Verlag, Berlin, 1995. 
[33] Jayasuriya, S. and M. Tomizuka, "Feedforward controllers for perfect tracking that does not invert plant zeros outside the unit disk," Proc. 12th IFAC World Congress, vol. 3, pp. 91-94, 1993.

[34] Kučera, V., Discrete Linear Control, John Wiley \& Sons, New York, 1979.

[35] Laschi, R., and G. Marro, "Alcune considerazioni sull'osservabilità dei sistemi dinamici con ingressi inaccessibili," Rendiconti della LXX Riunione Annuale AEI, paper 1-4-01, Rimini, Italy, 1969.

[36] Marro, G., "Controlled and conditioned invariants in the synthesis of unknown-input observers and inverse systems," Control and Cybernetics (Poland), vol. 2, no. 3/4, pp. 81-98, 1973.

[37] Marro, G. and M. Fantoni, "Using preaction with infinite or finite preview for perfect or almost perfect digital tracking," in press, Proc. MELECON'96, Bari, Italy, 1996.

[38] Marro, G. and A. Piazzi, "Feedback systems stabilizability in terms of invariant zeros." In A. Isidori and T. J. Tarn (Ed.), Systems. Models and Feedback: Theory and Applications. Birkhäuser series "Progress in Systems and Control Theory", Birkhäuser, Boston, 1992.

[39] Marro, G. and A. Piazzi, "Regulation without transients under large parameter jumps." Proc. 12th IFAC World Congress, vol. 4, pp. 23-26, 1993.

[40] Marro, G. and A. Piazzi, "A geometric approach to multivariable perfect tracking," Congress, San Francisco, 1996. in press, Proc. 13th IFAC World Congress, San Francisco, 1996.

[41] Middleton, R.H., and G.C. Goodwin, Digital Control and Estimation, Prentice Hall, Englewood Cliffs, N.J., 1990.

[42] Morse, A.S., "Output controllability and system synthesis," SIAM J. Control, vol. 9, pp. 143-148, 1971.

[43] Morse, A.S., "Structural invariants of linear multivariable systems," SIAM J. Control, vol. 11, pp. 446-465, 1973.

[44] Morse, A.S. and W.M. Wonham, "Status of noninteracting control," IEEE Trans. Autom. Contr., vol. AC-16, no. 6, pp. 568-581, 1971.

[45] Mosca, E, Optimal, Predictive, and Adaptive Control, Prentice Hall, Englewood Cliffs, N.J., 1995.

[46] Piazzi, A., and G. Marro, "The role of invariant zeros in multivariable system stability," Proceedings of the 1991 European Control Conference, Grenoble, 1991.

[47] Schumacher, J.M.H., "Regulator synthesis using ( $C, A, B)$-pairs," IEEE Trans. Autom. Contr., vol. AC-27, no. 6, pp. 1211-1221, 1982.

[48] Schumacher, J.M.H., "The algebraic regulator problem from the state-space point of view," Linear Algebra and its Applications, vol. 50, pp. 487-520, 1983. 
[49] Schumacher, J.M.H., "Almost stabilizability subspaces and high gain feedback," IEEE Trans. Autom. Contr., vol. AC-29, pp. 620-627, 1984.

[50] Weiland, S. and J.C. Willems, "Almost disturbance decoupling with internal stability," IEEE Trans. Autom. Contr., vol. 34, pp. 277-286, 1989.

[51] Willems, J.C., "Almost invariant subspaces: an approach to high gain feedback design Part I: Almost controlled invariant subspaces," IEEE Trans. Autom. Contr., vol. AC-26, no. 1, pp. 235-252, 1981.

[52] Willems, J.C., "Almost invariant subspaces: an approach to high gain feedback design Part II: Almost conditionally invariant subspaces," IEEE Trans. Autom. Contr., vol. AC-27, no. 5, pp. 1071-1085, 1982.

[53] Willems, J.C., and C. Commault, "Disturbance decoupling by measurement feedback with stability or pole placement," SIAM J. Contr. Optimiz., vol. 19, no. 4, pp. 490-504, 1981.

[54] Wonham, W.M., "Tracking and regulation in linear multivariable systems," SIAM J. Control, vol. 11, no. 3, pp. 424-437, 1973.

[55] Wonham, W.M., Linear Multivariable Control: A Geometric Approach, Springer-Verlag, New York, 1985.

[56] Wonham, W.M., "Geometric state-space theory in linear multivariable control: a status report," Automatica, vol. 15, pp. 5-13,1979.

[57] Wonham, W.M. and A.S. Morse, "Decoupling and pole assignment in linear multivariable systems: a geometric approach," SIAM J. Control, vol. 8, no. 1, pp. 1-18, 1970.

[58] Wonham, W.M., and J.B. Pearson, "Regulation and internal stabilization in linear multivariable systems," SIAM J. Control, vol. 12, no. 1, pp. 5-18, 1974. 